\title{
Relative rounding in toric and logarithmic geometry
}

\author{
CHIKARA NAKAYAMA \\ ARTHUR OGUS
}

\begin{abstract}
We show that the introduction of polar coordinates in toric geometry smoothes a wide class of equivariant mappings, rendering them locally trivial in the topological category. As a consequence, we show that the Betti realization of a smooth proper and exact mapping of log analytic spaces is a topological fibration, whose fibers are orientable manifolds (possibly with boundary). This turns out to be true even for certain noncoherent log structures, including some families familiar from mirror symmetry. The moment mapping plays a key role in our proof.
\end{abstract}

14D06, 14M25, 14F45, 32S30; 53D20, 14T05

\section{Introduction}

Our goal in this note is to study singularities of mappings of toric varieties, and more generally, logarithmic analytic spaces. We shall show that the introduction of polar coordinates - which effectuates a kind of real blowing up — smoothes out a wide class of such mappings, rendering them locally trivial (submersive) in the topological category. Suitably globalized, this technique provides a powerful tool for analyzing the geometry of degenerations. Some cohomological manifestations of this technique have already been studied by Kajiwara and Nakayama in [9], with applications to monodromy and vanishing cycles by Illusie, Kato and Nakayama in [7], and by Ogus in [15].

To give a flavor of our results, let us consider some simple examples. The most basic is the long-studied case of stable reduction. Let

$$
f: X=\mathbf{C} \times \mathbf{C} \rightarrow \mathbf{C}=S
$$

be the map sending $\left(x_{1}, x_{2}\right)$ to $t:=x_{1} x_{2}$. All the fibers $X_{t}$ for $t \neq 0$ are isomorphic, and in fact the entire family becomes trivial over $S^{*}:=S \backslash\{0\}$ : there is a commutative diagram

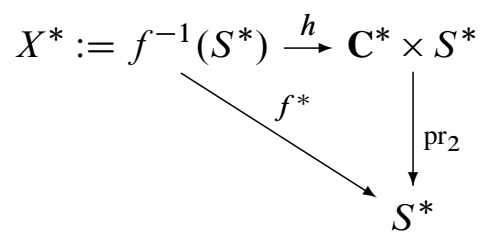


where $\mathrm{pr}_{2}$ is the projection to the second factor and $h$ is a homeomorphism, for example the map taking $\left(x_{1}, x_{2}\right)$ to $\left(x_{1}, x_{1} x_{2}\right)$. Such a trivialization cannot extend to the entire family over $S$, because the fiber $X_{0}=\left\{\left(x_{1}, x_{2}\right): x_{1} x_{2}=0\right\}$ is not homeomorphic to the "general" fibers $X_{t}$. The situation changes if one introduces polar coordinates. Let $\mathbf{R}_{\geq}:=\{r \in \mathbf{R}: r \geq 0\}$, let $\mathbf{S}^{1}:=\{\zeta \in \mathbf{C}:|\zeta|=1\}$, let $\mathbf{C}_{\log }:=\mathbf{R}_{\geq} \times \mathbf{S}^{1}$, and let $\tau: \mathbf{C}_{\log } \rightarrow \mathbf{C}$ be the map sending $(r, \zeta)$ to $r \zeta$. Note that $\tau$ is a proper surjective morphism; it is a real oriented blowup of the origin. (We will not need to define this notion precisely.) Then if $X_{\log }:=\mathbf{C}_{\log } \times \mathbf{C}_{\log }$, the map $f$ lifts naturally to $X_{\log }$, so that there is a commutative diagram:

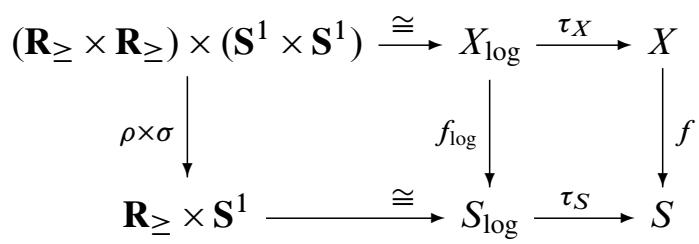

Here the map $\sigma: \mathbf{S}^{1} \times \mathbf{S}^{1} \rightarrow \mathbf{S}^{1}$ sends $\left(\zeta_{1}, \zeta_{2}\right) \rightarrow \zeta_{1} \zeta_{2}$ and can be trivialized in the same way as $f^{*}$. The map $\rho: \mathbf{R}_{\geq} \times \mathbf{R}_{\geq} \rightarrow \mathbf{R}_{\geq}$sends $\left(r_{1}, r_{2}\right) \rightarrow r_{1} r_{2}$. Its fiber over a nonzero $r \in \mathbf{R}_{\geq}$is homeomorphic to the set $\mathbf{R}_{>}$of positive numbers, and its fiber over zero consists of two copies of $\mathbf{R}_{\geq}$glued together at the origin. In fact this fiber is homeomorphic to all of $\mathbf{R}$ and hence to all the other fibers, and it is not difficult to write down a trivialization of the entire family $\rho$. This means that the blown-up family $f_{\log }: X_{\log } \rightarrow S_{\log }$ has become trivial. For a more complicated example, let $X:=\left\{\left(z_{1}, z_{2}, z_{3}, z_{4}\right) \in \mathbf{C}^{4}: z_{1} z_{2}=z_{3} z_{4}\right\}$ and let $f: X \rightarrow S:=\mathbf{C}$ be the function $z_{4}$. Then there is an evident polar blowup $f_{\log }: X_{\log } \rightarrow S_{\log }$, and it turns out that this too is, in the topological category, a trivial family, in which the fibers are topological manifolds with boundary. (A more subtle blowup allows one to obtain a family of manifolds without boundary.)

The examples above are equivariant mappings of affine toric varieties. Such mappings, to which our methods apply quite generally, serve as local models for many typical degenerations in algebraic geometry. The language of log geometry allows one to piece together these local models in a canonical way, thus justifying our study of equivariant mappings. As in the examples above, the main difficulty comes in the study of the "nonnegative real part" of such mappings.

Our main local result is most conveniently expressed in terms of constructions involving monoids. All monoids discussed in this paper will be commutative. A (commutative) monoid $Q$ is said to be fine if it is integral (ie, cancellative), and finitely generated (as a monoid), and to be sharp if its group of units $Q^{*}$ is trivial. We let $\left(\mathbf{R}_{\geq}, \cdot\right)$ denote the multiplicative monoid of nonnegative real numbers, endowed with its usual topology. 
Let $Q$ be a monoid and let $X_{Q}$ be the set of monoid homomorphisms from $Q$ to $\left(\mathbf{R}_{\geq}, \cdot\right)$. Each $q \in Q$ defines a function

$$
e_{q}: X_{Q} \rightarrow \mathbf{R}_{\geq}, \quad x \mapsto x(q),
$$

and we endow $X_{Q}$ with the weak topology and monoid structure defined by the set of such functions. The following result is a simple but important special case of our main theorem.

Proposition 0.1 Let $Q$ be a fine sharp monoid and let $p$ be a nonzero element of $Q$. Then the map $e_{p}: X_{Q} \rightarrow \mathbf{R}_{\geq}$is homeomorphic to a product map. That is, there exist a topological space $Z$ and a commutative diagram

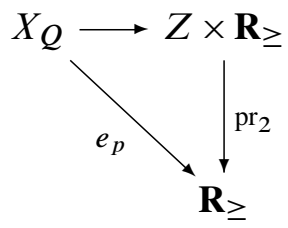

in which the horizontal map $X_{Q} \rightarrow Z \times \mathbf{R}_{\geq}$is a homeomorphism.

Somewhat more generally, let $V_{Q}:=\mathbf{R} \otimes Q^{\mathrm{gp}}$ and let $C_{Q}$ be the real subcone of $V_{Q}$ spanned by $Q$ (that is, the set of linear combinations of elements of $Q$ with nonnegative coefficients). Then each $c \in C_{Q}$ also defines a function $e_{c}: X_{Q} \rightarrow \mathbf{R}_{\geq}$, and in fact Proposition 0.1 is true for all nonzero $c \in C_{Q}$.

It is natural to ask if Proposition 0.1 holds more generally for a suitable class of morphisms $\theta: P \rightarrow Q$ of finitely generated monoids or cones. Some hypotheses are clearly required; for example, if $\theta: \mathbf{N}^{2} \rightarrow \mathbf{N}^{2}$ is the map sending $(a, b)$ to $(a, a+b)$, the dimension of the fibers of $X_{\theta}$ is not constant, so $X_{\theta}$ cannot be homeomorphic to a projection mapping. Our generalization of Proposition 0.1 depends on Kato's important notion of exactness. After a review of this notion and some of its variants (see Definition 2.1), we shall prove the following result.

Theorem 0.2 Let $P$ and $Q$ be fine monoids and let $\theta: C_{P} \rightarrow C_{Q}$ be an injective, exact, and locally exact morphism of their corresponding real cones. Then there exist a topological space $Z$ and a commutative diagram

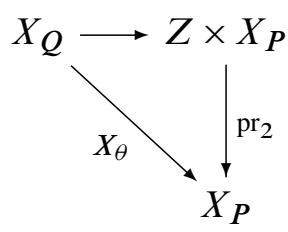

in which the horizontal map is a homeomorphism. 
This result affirmatively answers the question raised by Kajiwara and Nakayama in [ 9 , B.3] under the rubric "relative rounding," in the context of log geometry. As explained by Kato and Nakayama in [13], associated to an analytic space $X$ endowed with a log structure (satisfying suitable conditions which we relax here), there is a canonical surjective and proper map $\tau_{X}: X_{\log } \rightarrow X$, globalizing the construction using polar coordinates we saw in the initial examples. Then Theorem 0.2 and some standard arguments, which we shall review in Section 3, imply the following.

Theorem 0.3 Let $f: X \rightarrow Y$ be a smooth and exact morphism of fine log analytic spaces. Then the associated map of Betti realizations $f_{\log }: X_{\log } \rightarrow Y_{\log }$ is a topological submersion. That is, locally on $X_{\log }$ and $Y_{\log }$ it is homeomorphic to a projection from a product; furthermore the fibers are topological manifolds with boundary.

It follows (see Theorem 5.1) that if $f$ is exact, smooth, separated, and proper, then $f_{\log }$ is a "fiber bundle": locally on $Y_{\log }, f_{\log }: X_{\log } \rightarrow Y_{\log }$ is a projection from a product. This result can be used to give a new and more direct proof of some of the main theorems of Kajiwara and Nakayama [9] — for example, that the cohomology sheaves $R^{q} f_{\log *}(\mathbf{Z})$ are locally constant on $Y_{\log }$.

As we shall see in Theorem 3.7, many of these results apply even more generally, to some log structures which are not "coherent," but only "relatively coherent." Such log structures arise naturally in the study of some degenerations of Calabi Yau varieties and have been considered already by Ogus in [15] and Gross and Siebert in [4]. For example, our results hold for the Dwork family of K3 surfaces over $S:=\mathbf{C} \backslash \mu_{4}$ defined by $s\left(X_{0}^{4}+X_{1}^{4}+X_{2}^{4}+X_{3}^{4}\right)=4 X_{0} X_{1} X_{2} X_{3}, s \in S$, even though this family is not $d$-stable and falls outside the scope of the results of Kajiwara and Nakayama [9]. (This uses the "more subtle blowup" we referred to earlier.)

A key role in our proof is played by the moment map, which gives a linear description of the topological space $X_{Q}$. Let $S$ be any finite set of generators for a fine monoid $Q$. Then the associated moment map is defined by

$$
\mu_{S}: X_{Q} \rightarrow C_{Q}, \quad x \mapsto \sum_{s \in S} x(s) s .
$$

It is essentially proved in the textbook of Fulton [2, Section 4.2] that this map is a homeomorphism, compatible with the faces of $X_{Q}$ and $C_{Q}$ (see Theorem 1.4 for a more precise and general statement). The difficulty in applying this construction is that it is not functorial with respect to morphisms of monoids. However our methods show that a certain functoriality can be "forced," as explained in Proposition 2.11.

Our paper is organized as follows. Section 1 is a review of some basic facts about cones and monoids, including a new (and for us more conceptual) treatment of the 
moment mapping which will play a crucial role. The heart of our paper is Section 2, which contains the proof of Theorem 0.2. Section 3 contains a brief introduction to log geometry and the proof of Theorem 0.3 and its relatively coherent variant. Section 4 describes a generalization of our main results to "idealized" monoids and log analytic spaces, which can be useful in dealing with the strata that arise naturally from log structures. The last section is devoted to applications to cohomology, including a discussion of monodromy, orientation, and duality. There is also an appendix with an elementary proof that higher direct images of a locally constant sheaf by a separated proper submersion are again locally constant.

Many special cases and consequences of our results have been known for a long time. The case of semistable reduction for example, has a long history, going back at least to SGA 7 [5, Exposé I] (before the invention of log structures) and more recently was treated in the context of log geometry by Usui [20;19]. Cohomological and homotopical versions appear in works by Kajiwara and Nakayama [9] and Ogus [16; 15].

We are grateful to many mathematicians who helped and encouraged us in this work. Particular thanks go to Robion Kirby and Martin Olsson for many discussions, to Bernd Sturmfels for pointing us to the book Algebraic statistics for computational biology [17], and to Luc Illusie for his help and advice with Section 5. We are also very grateful to Ofer Gabber who pointed out a considerable simplification of our original argument, and to the referee for correcting many misprints.

\section{The moment map}

We begin by reviewing some well-known facts about the structures of $X_{Q}$ and $C_{Q}$ which will be important in the proofs and applications of our main results.

Recall that an ideal in a monoid $Q$ is a subset $J$ which is invariant under translation by elements of $Q$, and that an ideal is prime if its complement is a submonoid of $Q$. The submonoids $F$ of $Q$ whose complements are prime ideals are the faces of $Q$. If $F$ is a face of $Q$, then $q_{1}+q_{2}$ belongs to $F$ if and only if $q_{1}$ and $q_{2}$ belong to $F$. For $q \in Q$, we denote by $\langle q\rangle$ the smallest face of $Q$ containing $q$, ie, the set of all $f$ such that there exist $f^{\prime} \in Q$ and $n \in \mathbf{N}$ such that $f+f^{\prime}=n q$. If $h: Q \rightarrow Q^{\prime}$ is a homomorphism of monoids and $F^{\prime}$ is a face of $Q^{\prime}$, then $h^{-1}\left(F^{\prime}\right)$ is a face of $Q$. If $S$ is a subset of $Q$, we denote by $\lambda_{S}: Q \rightarrow Q_{S}$ the localization of $Q$ by $S$, ie, the universal map from $Q$ to a monoid in which the elements of $S$ become invertible. If $F$ is a face of $Q$, then $F=\lambda_{F}^{-1}\left(Q_{F}^{*}\right)$. A basic fact from duality theory for monoids asserts that if $F$ is a face of a fine monoid $Q$, there is a homomorphism $h: Q \rightarrow(\mathbf{N},+)$ such that $F=h^{-1}(0)$ [14]. 
Remark 1.1 Let $Q$ be an integral monoid, let $V_{Q}:=\mathbf{R} \otimes Q^{\mathrm{gp}}$, and let $C_{Q} \subseteq V_{Q}$ be the real cone spanned by $Q$, ie, the set of linear combinations of elements of $Q$ with nonnegative coefficients. Then the map $Q \rightarrow C_{Q}$ induces a bijection from the set of faces of $C_{Q}$ to the set of faces of $Q$. To check this, first note that we may assume without loss of generality that $Q^{\mathrm{gp}}$ is torsion free, since the map from $Q$ to its image $Q^{\prime}$ in $Q^{\mathrm{gp}} / Q_{\mathrm{tor}}^{\mathrm{gp}}$ induces a bijection $\operatorname{Spec} Q^{\prime} \rightarrow \operatorname{Spec} Q$ and an isomorphism $C_{Q} \rightarrow C_{Q^{\prime}}$. When $Q^{\mathrm{gp}}$ is torsion free, the map $Q \rightarrow C_{Q}$ is injective, and we write it as an inclusion. Note that if $G$ is a face of $C_{Q}$, then necessarily $G$ is invariant under the action of $\mathbf{R}_{\geq}$. Indeed, if $r \in \mathbf{R}_{\geq}$and $g \in G$, choose $n \in \mathbf{N}$ with $n \geq r$. Then $n g \in G$, and $n g=r g+(n-r) g$, hence $r g$ belongs to $G$. Now any $g \in G$ can be written $g=\sum r_{q} q$ with $r_{q} \in \mathbf{R}_{\geq}$and $q \in Q$, and it follows that $q \in G$ whenever $r_{q}>0$. Hence $G$ is the cone spanned by $G \cap Q$, a face of $Q$. Conversely, we claim that if $F$ is any face of $Q$, then $C_{F}$ is a face of $C_{Q}$ and $C_{F} \cap Q=F$. Suppose that $c=\sum r_{f} f$ and $c^{\prime}=\sum r_{f^{\prime}} f^{\prime}$ belong to $C_{Q}$ and $c+c^{\prime} \in C_{F}$. There is a fine submonoid $Q^{\prime}$ of $Q$ such that $C_{Q^{\prime}}$ contains all $f$ and $f^{\prime}$ with $r_{f}$ or $r_{f^{\prime}}$ nonzero. Choose a homomorphism $h: Q^{\prime} \rightarrow \mathbf{N}$ such that $h^{-1}(0)=F \cap Q^{\prime}$. Then $h$ induces a homomorphism $C_{h}: C_{Q^{\prime}} \rightarrow\left(\mathbf{R}_{\geq},+\right)$, and one sees immediately that $C_{h}^{-1}(0)=C_{F \cap Q^{\prime}}$. It follows that $C_{F \cap Q^{\prime}}$ is a face of $C_{Q^{\prime}}$ and hence that $c$ and $c^{\prime}$ belong to $C_{F \cap Q^{\prime}} \subseteq C_{F}$. Hence $C_{F}$ is a face of $C_{Q}$. Similarly, if $q \in Q \cap C_{F}$, say $q=\sum r_{f} f$ with $f \in F$, there is a fine submonoid $Q^{\prime}$ of $Q$ containing $q$ and all $f$ with $r_{f}>0$. Then choosing $h$ as above, we see that $h(q)=C_{h}(q)=0$, and hence $q \in F$.

An ideal $J$ of $C_{Q}$ is said to be a radical ideal if $r q \in J$ whenever $q \in J$ and $r>0$. Then if $c \in C_{Q} \backslash J$, it follows that $\langle c\rangle \subseteq C_{Q} \backslash J$. Hence $C_{Q} \backslash J$ is the union of the faces $F$ of $C_{Q}$ not meeting $J$, and $J$ is the intersection of the prime ideals which contain $J$.

If $\Sigma$ is a subset of $Q$, let $Z(\Sigma)$ be the set of $x \in X_{Q}$ which vanish on $\Sigma$. Then $Z(\Sigma)$ is closed ideal in the topological monoid $X_{Q}$, and $Z(\Sigma)=Z(I)$, where $I$ is the ideal of $Q$ generated by $\Sigma$. In fact the set of all subsets of the form $Z(I)$ defines another topology on $X_{Q}$, the Zariski topology. The irreducible closed sets for this topology are those defined by the prime ideals $\mathfrak{p}$ of $Q$. The complement $F$ of a prime ideal $\mathfrak{p}$ of $Q$ (resp. $C_{Q}$ ) is a face of $Q$ (resp. $C_{Q}$ ) and there is a natural embedding $i_{F}: X_{F} \rightarrow X_{Q}$, where

$$
i_{F}(x)(q):= \begin{cases}x(q) & \text { if } q \in F, \\ 0 & \text { otherwise. }\end{cases}
$$

The image of $i_{F}$ is precisely $Z(\mathfrak{p})$ and we will allow ourselves to identify $X_{F}$ with $Z(\mathfrak{p}) \subseteq X_{Q}$. Then we have identifications

$$
X_{F}^{*}:=X_{F \mathrm{gp}}=\left\{x \in X_{Q}: x^{-1}\left(\mathbf{R}_{\geq}^{*}\right)=F\right\},
$$


and $X_{Q}$ is the disjoint union $X_{Q}=\cup X_{F}^{*}$ as $F$ ranges over the faces of $Q$. For each $x \in X_{Q}$, let $F(x):=\{q \in Q: x(q) \neq 0\}$, the face of $Q$ such that $x \in X_{F(x)}^{*}$.

Observe that if $S$ is any finite set of generators of $C_{Q}$, the map $X_{Q} \rightarrow \mathbf{R}^{S}$ sending $x$ to the sequence $(x(s): s \in S)$ is injective and that its image is a closed subset of $\mathbf{R}^{S}$; furthermore $X_{Q}$ has the topology and monoid structure induced from $\mathbf{R}^{S}$.

A morphism $\theta: C_{P} \rightarrow C_{Q}$ of finitely generated cones induces a morphism

$$
X_{\theta}: X_{Q} \rightarrow X_{P}, \quad x \mapsto x \circ \theta .
$$

Then $X_{\theta}$ is continuous with respect to the standard topology and the Zariski topology.

The subset

$$
X_{Q}^{*}:=\operatorname{Hom}\left(Q, \mathbf{R}_{\geq}^{*}\right)=\operatorname{Hom}\left(Q^{\mathrm{gp}}, \mathbf{R}_{\geq}\right)
$$

is a submonoid of $X_{Q}$ and in fact is a topological group, isomorphic to a product of copies of $\left(\mathbf{R}_{\geq}^{*}, \cdot\right)$ (which in turn is isomorphic to the topological group $(\mathbf{R},+$ ) via the logarithm map). It acts naturally on $X_{Q}$, and each $X_{F}^{*}$ is stable under this action. In fact each $X_{F}^{*}$ is also naturally a Lie group. If $f \in F$ and $x \in X_{Q}, e_{f}(x)>0$ if $x \in X_{F}^{*}$. Then $\log \left(e_{f}\right)$ is a well-defined function on $X_{F}^{*}$, and its differential $d \log \left(e_{f}\right)$ is an invariant differential form. Then $f \mapsto d \log \left(e_{f}\right)$ induces a natural isomorphism from $V_{F}:=\mathbf{R} \otimes F^{\mathrm{gp}}$ to the space of invariant differential forms on $X_{F}^{*}$, and hence an isomorphism from the Lie algebra of $X_{F}^{*}$ to $V_{F}^{\vee}:=\operatorname{Hom}\left(F^{\mathrm{gp}}, \mathbf{R}\right)$. To simplify the notation we write these isomorphisms as identifications. Thus if $f \in F$, we view $1 \otimes f \in V_{F}$ as an invariant differential form on $X_{F}^{*}$, and if $\phi \in V_{F}^{\vee}$ we view $\phi$ as an invariant vector field on $X_{F}^{*}$. With this notation, we have the formula

$$
d e_{f}=e_{f} \otimes f .
$$

Similarly, the interior $C_{F}^{o}$ of the cone spanned by $F$ has a natural structure of a $C^{\infty}$ manifold, induced from the inclusion $C_{F}^{o} \subseteq V_{F}$, and the invariant vector fields on the ambient space $V_{F}$ are naturally identified with elements of $V_{F}$.

Let $\mathbf{R}[Q]$ (resp. $\mathbf{R}\left[C_{Q}\right]$ ) be the real monoid algebra of $Q$ (resp. $C_{Q}$ ). Its underlying vector space is just the set of real linear combinations of elements of $Q$ (resp. $C_{Q}$ ), which we also refer to as cycles in $Q$ (resp. $C_{Q}$ ). We say a cycle is effective if its coefficients are all nonnegative; the set of effective cycles is a submonoid of $\mathbf{R}[Q]$ (resp. $\mathbf{R}\left[C_{Q}\right]$ ) under addition (and multiplication). Each cycle $A:=\sum a_{q} q$ of $\mathbf{R}\left[C_{Q}\right]$ defines a function $e_{A}: X_{Q} \rightarrow \mathbf{R}_{\geq}$, where $e_{A}(x)=\sum a_{q} e_{q}(x): q \in C_{Q}$.

Definition 1.2 The moment map of an effective cycle $A$ in $C_{Q}$ is the function $X_{Q} \rightarrow$ $C_{Q}$ defined by

$$
\mu_{A}(x):=\sum_{q \in C_{Q}} a_{q} x(q) q
$$


An effective cycle $A$ in $C_{Q}$ can be viewed as a function $C_{Q} \rightarrow \mathbf{R}_{\geq}$with finite support. Thus if $F$ is a face of $Q$, the restriction $A_{\left.\right|_{F}}$ of $A$ to $C_{F}$ can be viewed as an effective cycle in $C_{F}$. If $x \in X_{F} \subseteq X_{Q}$, then $e_{q}(x)=0$ if $q \notin F$, so $e_{A} \circ i_{F}=e_{A_{\left.\right|_{F}}}$. Furthermore,

$$
\mu_{A}(x)=\sum_{q \in C_{Q}} a_{q} x(q) q=\sum_{f \in C_{F}} a_{f} x(f)
$$

and the following diagram commutes.

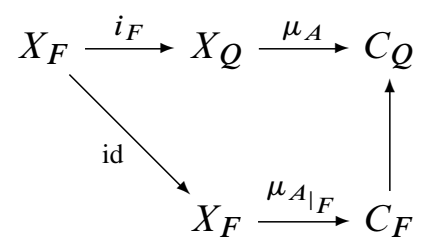

This will allow us to identify $e_{A_{\left.\right|_{F}}}$ with $e_{\left.A\right|_{X_{F}}}$ and $\mu_{A_{\mid}}$with $\mu_{\left.A\right|_{X_{F}}}$. Note that if the support $S$ of $A$ generates $C_{Q}$, then $S \cap C_{F}$ necessarily generates $C_{F}$, and if $x \in X_{F}$ (resp. $X_{F}^{*}$ ), then $\mu_{A}(x)$ belongs to $C_{F}$ (resp. $C_{F}^{o}$ ).

The following result describes the differential properties of the moment map.

Proposition 1.3 Let $A$ be an effective cycle in $C_{Q}$, let $S \subseteq C_{Q}$ be its support, and let $F$ be a face of $Q$.

(1) The restriction of the moment map $\mu_{A}$ to $X_{F}^{*}$ is the differential of the restriction of the function $e_{A}$ to $X_{F}^{*}$.

(2) Let $x$ be a point of $X_{F}^{*}$ and consider the derivative of $\mu_{A}$ at $x$ :

$$
\tau_{x}:=T_{x}\left(\mu_{A}\right): T_{x}\left(X_{F}^{*}\right) \rightarrow T_{\mu_{A}(x)}\left(C_{F}^{o}\right) \quad \text { ie, } \quad V_{F}^{\vee} \rightarrow V_{F} .
$$

Then the associated bilinear form $\beta_{x}$ on $V_{F}^{\vee}$ :

$$
\beta_{x}(\phi, \psi):=\psi\left(\tau_{x}(\phi)\right)
$$

is symmetric and positive semidefinite. If $S \cap C_{F}$ generates $C_{F}$, then $\beta_{x}$ is positive definite and $\tau_{x}$ is an isomorphism.

Proof As we have seen,

$$
e_{A} \circ i_{F}=\sum_{f \in F \cap S} a_{f} e_{f},
$$

so by the formula (1) for $d e_{f}$ on $X_{F}^{*}$,

$$
d e_{A_{\mid} F}=\sum_{f \in F \cap S} a_{f} d e_{f}=\sum_{f \in F \cap S} a_{f} e_{f} \otimes f=\mu_{A_{\mid F}} .
$$


Then

$$
T_{x}\left(\mu_{A}\right)=\sum a_{f} d e_{f} \otimes f=\sum a_{f} e_{f} \otimes f \otimes f \in \mathbf{R}\left[C_{F}\right] \otimes \operatorname{Hom}\left(V_{F}^{\vee}, V_{F}\right) .
$$

In particular, for $x \in X_{F}^{*}$ and $\phi \in V_{F}^{\vee}$,

and

$$
\begin{aligned}
\tau_{x}(\phi) & =\sum_{f} a_{f} x(f) \phi(f) f \\
\beta_{x}(\phi, \psi) & =\sum_{f} a_{f} x(f) \phi(f) \psi(f) .
\end{aligned}
$$

Thus $\beta_{x}$ is symmetric. Since $a_{f}$ and $x(f)$ are nonnegative, $\beta_{x}$ is also positive semidefinite. If $S \cap C_{F}$ generates $C_{F}$, then $S \cap C_{F}$ spans $V_{F}$, so $\beta_{x}$ is positive definite and consequently $\tau_{x}$ is an isomorphism.

The following result is well-known. We present a proof based on a combination of ideas from Fulton [2] and Kajiwara and Nakayama [9, A.1.1], with a simplification suggested by Gabber.

Theorem 1.4 Let $Q$ be a fine monoid and $A$ an effective cycle in $C_{Q}$ whose support generates $C_{Q}$. The moment map $\mu_{A}$ is a homeomorphism

$$
\mu_{A}: X_{Q} \rightarrow C_{Q}
$$

compatible with the stratifications of $X_{Q}$ and $C_{Q}$ induced by the faces of $Q$.

Proof We have already observed that if $F$ is a face of $Q, \mu_{A}$ maps $X_{F}$ to $C_{F}$ and $X_{F}^{*}$ to $C_{F}^{o}$, so that $\mu_{A}$ is compatible with the stratifications by faces. Thus to prove that $\mu_{A}$ is injective it will suffice to prove that for each face $F, \mu_{A}$ induces an injection $X_{F}^{*} \rightarrow C_{F}^{o}$. To simplify notation, we may and shall assume that $F=Q$. We have an isomorphism

$$
\exp _{Q}: V_{Q}^{\vee} \rightarrow X_{Q}^{*}, \quad \phi \mapsto \exp \circ \phi .
$$

If $\phi$ and $\phi^{\prime}$ are distinct points of $V_{Q}^{\vee}$, let $\psi:=\phi^{\prime}-\phi$, and for each real number $t$, let $\phi_{t}:=\phi+t \psi$ and $x_{t}=\exp _{Q}\left(\phi_{t}\right)$. Then it follows from (2) of Proposition 1.3 that the derivative of $\psi\left(\mu_{A}\left(x_{t}\right)\right)$ with respect to $t$ is $\beta_{x_{t}}(\psi, \psi)>0$. Explicitly,

$$
\psi\left(\mu_{A}\left(x_{t}\right)\right)=\sum_{s} a_{s} \psi(s) \exp (\phi(s)+t \psi(s)),
$$

and the derivative with respect to $t$ is

$$
\sum_{s} a_{s}(\psi(s))^{2} x_{t}(s)>0
$$

Thus $\psi\left(\mu_{A}\left(x_{t}\right)\right)$ is an increasing and hence injective function of $t$. This implies that $\mu_{A}(\exp \circ \phi) \neq \mu_{A}\left(\exp \circ \phi^{\prime}\right)$. 
Lemma 1.5 If the support $S$ of $A$ generates $C_{Q}$, then $\mu_{A}: X_{Q} \rightarrow C_{Q}$ is proper.

Proof We assume without loss of generality that $0 \notin S$. If $S$ generates $C_{Q}$, then the evaluation map $X_{Q} \rightarrow \mathbf{R}^{S}$ is a closed immersion. Thus a closed subset of $X_{Q}$ is compact if its image in $\mathbf{R}^{S}$ is bounded, and it will suffice to prove that $\mu_{A}^{-1}(B)$ is bounded if $B$ is. This is easy to verify if $C_{Q}$ is sharp. In this case, we can choose a local homomorphism $h: C_{Q} \rightarrow \mathbf{R}_{\geq}$. Then if $B$ is a bounded subset of $C_{Q}, h(B)$ is a bounded subset of $\mathbf{R}_{\geq}$, say bounded by $M$. If $x \in \mu_{A}^{-1}(B)$, then

$$
M \geq h\left(\mu_{A}(x)\right)=\sum_{s} a_{s} h(s) x(s),
$$

and hence each $x(s) \leq M / a m$, where $a$ is the minimum of $\{a(s): s \in S\}$ and $m$ is the minimum of $\{h(s): s \in S\}$.

For the general case, we argue as follows. ${ }^{1}$ Let $\Sigma$ denote the set of all subsets $\sigma$ of $S$ such that the subcone $C_{\sigma}$ of $C_{Q}$ generated by $\sigma$ is sharp. For each such $\sigma$, we can choose a local homomorphism $h_{\sigma}: C_{\sigma} \rightarrow \mathbf{R}_{\geq}$, which we can then extend to a linear map $V_{Q} \rightarrow \mathbf{R}$. Since $\Sigma$ is finite, we can choose an $m>0$ such that $h_{\sigma}(s)>m$ for all $\sigma \in \Sigma$ and $s \in \sigma$. If $x \in X_{Q}$, let $\sigma_{x}:=\{s \in S: x(s) \geq 1\}$. Then $x$ defines a homomorphism $C_{\sigma_{x}} \rightarrow\left(\mathbf{R}_{>}, \cdot\right)$ and hence $\log x$ is a homomorphism $C_{\sigma_{x}} \rightarrow(\mathbf{R},+)$ which is nonnegative on each element of $\sigma_{x}$. It follows that $C_{\sigma_{x}}$ is sharp, so that $\sigma_{x} \in \Sigma$. Now if $B$ is a bounded subset of $C_{Q}$, there is a bound $M$ for $\cup\left\{h_{\sigma}(B): \sigma \in \Sigma\right\}$. Then if $x \in \mu_{A}^{-1}(B)$,

$$
M \geq h_{\sigma_{x}}\left(\mu_{A}(x)\right)=\sum_{s \in \sigma_{x}} a_{s} h_{\sigma_{x}}(s) x(s)+\sum_{s \notin \sigma_{x}} a_{s} h_{\sigma_{x}}(s) x(s) .
$$

The second sum need not be positive, but its absolute value is bounded by $c:=$ $\sum_{s} a_{s}\left|h_{\sigma_{x}}(s)\right|$. Thus for $s \in \sigma_{x}, a_{s} h_{\sigma_{x}} x(s) \leq M+c$ and hence $x(s) \leq(M+c) / a m$. On the other hand, for $s \notin \sigma_{x}, x(s) \leq 1$. It follows that $\mu_{A}^{-1}(B)$ is bounded.

It is now easy to see that, when $S$ generates $C_{Q}, \mu_{A}$ is a homeomorphism. For each face $F$ of $Q, X_{F}^{*}=\mu_{A}^{-1}\left(C_{F}^{o}\right)$, and in particular $\mu_{A}$ induces a proper map $\mu_{A, F}: X_{F}^{*} \rightarrow C_{F}^{o}$. As we have seen in Proposition 1.3, $\mu_{A, F}$ is a differentiable map of differentiable manifolds which induces an isomorphism on tangent spaces at every point, and it follows from the implicit function theorem that its image is open. Since it is also proper, its image is also closed, and since $C_{F}^{o}$ is connected, it follows that $\mu_{A, F}$ is surjective. We conclude that $\mu_{A}$ is bijective, continuous, and proper, and it follows that it is a homeomorphism, since a proper map of Hausdorff spaces is closed.

${ }^{1}$ This argument, more direct than our original one, is due to Gabber. 
Remark 1.6 The simple proof of surjectivity we explained above is due to Gabber. Our original argument proved surjectivity directly, without using the properness of $\mu_{A}$, which we proved after the surjectivity. Since this argument is also short and perhaps interesting, we reproduce it here. This proof is inspired by the discussion of Birch's theorem [17, Theorem 1.10]; we thank Bernd Sturmfels for alerting us to this paper.

Let $c$ be an interior point of $C_{Q}$. To prove that $c$ lies in the image of $\mu_{A}$, let

$$
\Psi_{c}:=e_{c} \exp \left(-e_{A}\right): X_{Q} \rightarrow \mathbf{R}_{\geq} .
$$

Lemma 1.7 For every positive number $r$, the set $X_{A, c}(r)$ of all $x \in X_{Q}$ such that $\Psi_{c}(x) \geq r$ is compact and contained in $X_{Q}^{*}$.

Proof Let $S$ be the support of $A$. Since $c$ is in the interior of $C_{Q}$, it is not contained in any proper face. Thus for every $s \in S$, there exist $n_{s} \in \mathbf{Z}_{>}$and $q_{s} \in C_{Q}$ with $n_{s} c=s+q_{s}$, so $n_{s} c=s+\sum_{t \in S} a_{t} t$ with all $a_{t} \in \mathbf{R}_{\geq}$. Summing over $s \in S$ and dividing by $\sum n_{s}$, we find that $c=\sum\left\{c_{s} s: s \in S\right\}$ with each $c_{s}>0$. If $F$ is a proper face of $Q$ and $x \in X_{F}$, then $x(s)=0$ for some $s \in S$, hence $e_{c}(x)=\prod x(s)^{c_{s}}=0$. Thus $\Psi_{c}$ vanishes on $X_{Q} \backslash X_{Q}^{*}$. It follows that $X_{A, c}(r) \subseteq X_{Q}^{*}$ when $r>0$. Since $X_{A, c}(r)$ is a closed subset of a Euclidean space, its compactness will follow if we prove it is bounded. Since $X_{A, c}(r)$ is contained in $X_{Q}^{*}$, we can let $\psi:=-\log \Psi_{c}$, and it suffices to prove that for any real number $m$, the set $X_{A, c}^{*}(m)$ of points of $X_{Q}^{*}$ where $\psi<m$ is bounded. For $x \in X_{Q}^{*}$,

$$
\psi(x)=e_{A}(x)-\log e_{c}(x)=\sum_{s}\left(a_{s} x(s)-c_{s} \log x(s)\right)=\sum_{s} \psi_{s}(x(s)),
$$

where $\psi_{s}(t):=a_{s} t-c_{s} \log t$. Since $a_{s}$ and $c_{s}$ are both nonnegative, the function $\psi_{s}$ is bounded below, and we can find a positive number $m^{\prime}$ such that $\psi_{s}(t) \geq-m^{\prime}$ for all $t$ and all $s$. If $x \in X_{A, c}^{*}(m)$ we have, for each $s \in S$,

$$
\psi_{s}(x(s))=\psi(x)-\sum_{s^{\prime} \neq s} \psi_{s^{\prime}}\left(x\left(s^{\prime}\right)\right) \leq \psi(x)+|S| m^{\prime} \leq m^{\prime \prime}:=m+|S| m^{\prime} .
$$

On the other hand, the restriction of $\psi_{s}$ to $\left[c_{s} / a_{s}, \infty\right)$ is an increasing function which tends to $\infty$. Hence for each $s$, there is a constant $m_{s}$ such that $t<m_{s}$ whenever $\psi_{s}(t)<m^{\prime \prime}$. Then if $x \in X_{A, c}^{*}(m)$, each $x(s)<m_{s}$. This shows that $X_{A, c}^{*}(m)$ is indeed bounded.

Now choose a point $x_{0}$ in $X_{Q}^{*}$. Then $r:=\Psi_{c}\left(x_{0}\right)>0$, and the set $X_{A, c}(r / 2)$ is nonempty and compact. It follows that $\Psi_{c}$ has a maximum at some point $x$ of 
$X_{A, c}(r / 2)$. But then in fact $\Psi_{c}(x)$ is the maximum of $\Psi_{c}$ on all of $X_{Q}^{*}$. Thus $\Psi_{c}$ has a critical point at $x$, and hence so does $\log \Psi_{c}$. So $d \log \Psi_{c}(x)=0$. By Proposition 1.3,

$$
d \log \Psi_{c}=d \log e_{c}-d e_{A}=c-\mu_{A},
$$

thus $\mu_{A}(x)=c$.

\section{Relative rounding}

This section is devoted to a precise formulation and proof of Theorem 0.2. We begin by reviewing the notion and properties of exactness. The main ideas are taken from the appendix of Illusie, Kato and Nakayama [7].

Definition 2.1 A morphism $\theta: P \rightarrow Q$ of integral monoids is

(1) local if $\theta^{-1}\left(Q^{*}\right)=P^{*}$,

(2) exact if the diagram

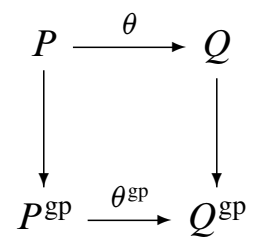

is Cartesian,

(3) locally exact if for every face $G$ of $Q$, the localized map

$$
P_{\theta^{-1}(G)} \rightarrow Q_{G}
$$

is exact,

(4) very locally exact if for every face $G$ of $Q$ such that $\theta^{-1}(G)=P^{*}$, the map

$$
P \rightarrow Q_{G}
$$

is exact.

Remark 2.2 If $P=\mathbf{N}$, then $\theta$ is exact if and only if it is local. In general, an exact morphism is easily seen to be local, so a locally exact morphism is exact if and only if it is local. Furthermore, if $\theta$ is exact and $F$ is any face of $P$, then the induced map $P_{F} \rightarrow Q_{F}$ is again exact, hence local, and it follows that there is a face $G$ of $Q$ such that $\theta^{-1}(G)=F$. Thus if $\theta$ is exact, $\operatorname{Spec}(\theta)$ is surjective. For fine saturated monoids the converse is also true: if $P$ and $Q$ are fine saturated monoids, then $\theta$ is exact if and only if $\operatorname{Spec} \theta$ is surjective [7, A.3.2.1]. Moreover, if $P$ and $Q$ are fine sharp monoids and $\theta: C_{P} \rightarrow C_{Q}$ is injective, then $\theta$ is locally exact if and only if the map on monoid algebras $\mathbf{Z}\left[C_{P}\right] \rightarrow \mathbf{Z}\left[C_{Q}\right]$ is flat. One direction follows, for example, 
from Theorem 2.3 below. See Kato [11] for the other direction as well as the relation between local exactness to Kato's notion of integrality for morphisms of monoids.

Theorem 2.3 Let $P$ and $Q$ be fine monoids and let $\theta: C_{P} \rightarrow C_{Q}$ be an injective and local homomorphism of their corresponding real cones. Assume $P$ is sharp. Then the following conditions are equivalent.

(1) $\theta$ is locally exact.

(2) $\theta$ is very locally exact.

(3) For each $q \in C_{Q}$,

$$
S_{q}:=\left(q+\theta\left(C_{P}^{\mathrm{gp}}\right)\right) \cap C_{Q}
$$

is isomorphic as a $C_{P}$-set to $C_{P}$, generated by a unique $q_{0} \in C_{Q}$ with the property that $\theta^{-1}\left\langle q_{0}\right\rangle=\{0\}$.

Proof In what follows we regard $C_{P}$ as a subcone of $C_{Q}$. Observe first that, in any case, $\left\{S_{q}: q \in C_{Q}\right\}$ forms a partition of $C_{Q}$. We claim that each $S_{q}$ contains some $q_{0}$ such that $\left\langle q_{0}\right\rangle \cap C_{P}=\{0\}$. Choose a local homomorphism $h: C_{Q} \rightarrow\left(\mathbf{R}_{\geq},+\right)$. Then $h$ factors through a local homomorphism $\bar{h}: C_{\bar{Q}} \rightarrow\left(\mathbf{R}_{\geq},+\right)$, where $\bar{Q}:=Q / Q^{*}$ is sharp. One verifies immediately that the image of $S_{q}$ in $C_{\bar{Q}}$ is exactly $S_{\bar{q}}$. Since $\bar{Q}$ is sharp, $\bar{h}^{-1}[0, \bar{h}(\bar{q})]$ is compact, and since $S_{\bar{q}}$ is closed in $C_{\bar{Q}}$, its intersection with $\bar{h}^{-1}[0, \bar{h}(\bar{q})]$ is also compact. It follows that $\bar{h}$ achieves a minimum value on $S_{\bar{q}}$, say at $\bar{q}_{0}$, where $q_{0} \in S_{q}$. Suppose that $p \in\left\langle q_{0}\right\rangle \cap C_{P}$, so that there exists some $q^{\prime} \in C_{Q}$ such that $q_{0}=q^{\prime}+p$. Then $q^{\prime} \in S_{q_{0}}=S_{q}$, and $h\left(q_{0}\right)=h\left(q^{\prime}\right)+h(p) \geq h\left(q^{\prime}\right)$. Since $h\left(q^{\prime}\right) \geq h\left(q_{0}\right)$, it follows that $h(p)=0$, and since $\theta$ and $h$ are local and $P$ is sharp, it follows that $p=0$. Thus $\left\langle q_{0}\right\rangle \cap C_{P}=\{0\}$ as desired. Now if (2) holds, the map $C_{P} \rightarrow C_{Q}$ remains exact after localizing by $q_{0}$. Since $q_{0} \in S_{q}$, there exist $p$ and $p^{\prime} \in C_{P}$ such that $q+p=q_{0}+p^{\prime}$. Thus $p^{\prime}-p=q-q_{0}$ lies in the localization of $C_{Q}$ by $q_{0}$, and hence $p^{\prime}-p \in C_{P}$. It follows that $q=p^{\prime}-p+q_{0}$ lies in the $C_{P}$ orbit of $q_{0}$. Since $q \in S_{q}$ was arbitrary, we see that $q_{0}$ generates $S_{q}$ as a $C_{P}$-set. Now if $q_{1}$ is another element of $S_{q}$ such that $\left\langle q_{1}\right\rangle \cap C_{P}=\{0\}$, we have $q_{1}=q_{0}+p$ and $q_{0}=q_{1}+p^{\prime}$ for some $p, p^{\prime} \in C_{P}$. Since $C_{Q}$ is integral, this implies that $p+p^{\prime}=0$, and since $P$ is sharp, it follows that $p=p^{\prime}=0$ and that $q_{0}=q_{1}$. This proves that (2) implies (3).

Now suppose that (3) holds. Let $G$ be a face of $Q$ and let $F$ be its intersection with $P$. We will prove that $\left(C_{P}\right)_{F} \rightarrow\left(C_{Q}\right)_{G}$ is exact. Suppose that $p_{2}-p_{1}$ lies in the localization of $C_{Q}$ by $G$. Then there exist $q \in Q$ and $g \in G$ such that $p_{2}-p_{1}=q-g$. This implies that $S_{g}=S_{q}$. Condition (3) implies that there exists some $q_{0} \in C_{Q}$ which generates $S_{g}=S_{q}$ as a $C_{P}$-set. Thus there are $p, p^{\prime} \in C_{P}$ such that $g=p+q_{0}$ and 
$q=p^{\prime}+q_{0}$. Then $p_{2}-p_{1}=p^{\prime}-p$. On the other hand, $p \in\langle g\rangle \subseteq G$, so $p \in F$, so indeed $p_{2}-p_{1} \in\left(C_{P}\right)_{F}$, as required. This proves that (3) implies (1). The remaining implication is trivial.

The following consequence is a key ingredient in our proof of relative rounding. See Illusie, Kato and Nakayama [7, A.3.2.2].

Corollary 2.4 Let $P$ and $Q$ be fine monoids and let $\theta: C_{P} \rightarrow C_{Q}$ be a local and locally exact morphism of the corresponding cones. Assume that $P$ is sharp, and let $C_{Q, P} \subseteq C_{Q}$ denote the union of the set of faces $G$ of $C_{Q}$ such that $G \cap C_{P}=\{0\}$. Then the map

$$
g: C_{Q, P} \times C_{P} \rightarrow C_{Q}, \quad(q, p) \mapsto q+p
$$

is a homeomorphism, whose inverse we denote by

$$
\left(\pi_{Q, P}, \pi_{P}\right): C_{Q} \rightarrow C_{Q, P} \times C_{P} .
$$

Proof Since $\theta$ is local and locally exact, it is exact and therefore injective, since $P$ is sharp. It follows immediately from condition (3) of Theorem 2.3 that the map $g$ is bijective. It is clear that $g$ is continuous, and we claim it is also proper, hence a homeomorphism. It will suffice to prove that if $B$ is a subset of $C_{Q, P} \times C_{P}$ such that $B^{\prime}:=g(B) \subseteq C_{Q}$ is bounded, then $B$ is also bounded. Let $G$ be a face of $C_{Q}$ which intersects $C_{P}$ trivially, and let $h: C_{Q} \rightarrow\left(\mathbf{R}_{\geq},+\right)$be a homomorphism such that $h^{-1}(0)=G$. Let $B_{G}$ be the subset of $B$ consisting of those pairs $(q, p)$ such that $q \in G$, let $B_{G}^{\prime}:=g\left(B_{G}\right)$ and let $B_{G}^{\prime \prime}$ be the image of $B_{G}$ under the projection $C_{Q, P} \times C_{P} \rightarrow C_{P}$. Since $B_{G}^{\prime} \subseteq B^{\prime}$ is bounded, so is $h\left(B_{G}^{\prime}\right)$. If $(q, p) \in B_{G}$, $h(p)=h(q+p) \in h\left(B_{G}^{\prime}\right)$, and it follows that $h\left(B_{G}^{\prime \prime}\right)$ is bounded. Since the restriction of $h$ to $C_{P}$ is local and $P$ is sharp, it follows that $B_{G}^{\prime \prime}$ is also bounded. Repeating this for each face $G$ of $Q$ with $G \cap C_{P}=0$, we conclude that the projection of $B$ to $C_{P}$ is bounded. Since we also know that $B^{\prime}$ is bounded, it follows that the projection of $B$ to $C_{Q, P}$ is also bounded. Hence $B$ is bounded.

Our strategy for the proof of Theorem 0.2 will be to use moment maps to compare the spaces $X_{Q}$ and $X_{P}$ to the corresponding cones $C_{Q}$ and $C_{P}$ and then apply Corollary 2.4. This is not straightforward because the moment map is not functorial. Overcoming this difficulty is the main content of the rest of this section.

If $P$ is any monoid, let $P^{+}:=P \backslash P^{*}$ denote its maximal ideal. The vertex of $X_{P}$ is the element of $X_{P}$ defined by

$$
v_{P}(p):= \begin{cases}0 & \text { if } p \in P^{+} \\ 1 & \text { if } p \in P^{*}\end{cases}
$$


If $\theta: C_{P} \rightarrow C_{Q}$ is a morphism of cones, let $X_{Q, P}:=X_{\theta}^{-1}\left(Z\left(P^{+}\right)\right)$, ie, the Zariski closed subset of $X_{Q}$ defined by the ideal of $C_{Q}$ generated by $\theta\left(P^{+}\right)$. When $P$ is sharp, $X_{Q, P}=X_{\theta}^{-1}\left(v_{P}\right)$. Let $J$ be the radical of this ideal. Then $X_{Q, P}=Z(J)$, and $J$ is the intersection of the prime ideals of $C_{Q}$ containing $C_{P}^{+}$, ie, the prime ideals corresponding to the faces $G$ of $C_{Q}$ such that $\theta^{-1}(G)=C_{P}^{*}$ (see the sentence following Remark 1.1).

Let $A$ be an effective cycle in $C_{Q}$ whose support generates $C_{Q}$, and let $\mu_{A}: X_{Q} \rightarrow$ $C_{Q}$ be the associated moment map 1.2. We know from Theorem 1.4 that $\mu_{A}$ is a homeomorphism compatible with the stratifications by faces. Then its inverse $v_{A}$ enjoys the same property. Thus $\mu_{A}\left(X_{Q, P}\right)=C_{Q, P} \subseteq C_{Q}$ and $v_{A}$ induces a homeomorphism $v_{A, P}: C_{Q, P} \rightarrow X_{Q, P}$. We obtain a map $X_{Q} \rightarrow X_{Q, P}$ which will allow us to push the general fibers of $X_{\theta}$ to the special fiber. Figure 1 illustrates this map when $\theta$ is the diagonal embedding $\mathbf{N} \rightarrow \mathbf{N} \oplus \mathbf{N}$ and $A$ is the minimal set of generators of $\mathbf{N} \oplus \mathbf{N}$; in this case $\mu_{A}$ is the identity map of $\mathbf{R}_{\geq} \times \mathbf{R}_{\geq}$.

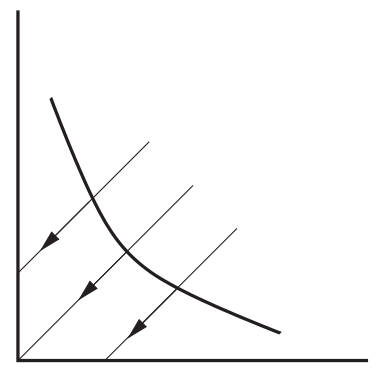

Figure 1

Theorem 2.5 Let $P$ and $Q$ be fine monoids, with $P$ sharp, and let $\theta: C_{P} \rightarrow C_{Q}$ be a local and locally exact morphism of their corresponding real cones. Let $A$ be an effective generating cycle of $C_{Q}$ and let $\eta_{A, P}$ be the composite

$$
\eta_{A, P}: X_{Q} \stackrel{\mu_{A}}{\longrightarrow} C_{Q} \stackrel{\pi_{Q, P}}{\longrightarrow} C_{Q, P} \stackrel{\nu_{A, P}}{\longrightarrow} X_{Q, P},
$$

where $\pi_{Q, P}: C_{Q} \rightarrow C_{Q, P}$ is the map defined in Corollary 2.4. Then in the commutative diagram

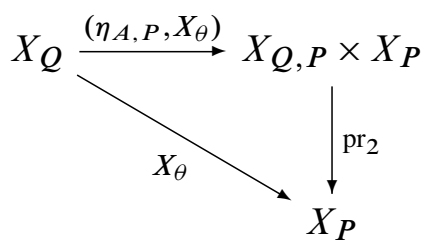

the horizontal arrow $\left(\eta_{A, P}, X_{\theta}\right)$ is a homeomorphism. 
Remark 2.6 The map $\eta_{A, P}: X_{Q} \rightarrow X_{Q, P}$ has the following property. For each $x \in X_{Q}, F(x)$ is the face of $C_{Q}$ generated by $F\left(\eta_{A}, P(x)\right)$ and $F(x) \cap C_{P}$, and, consequently, the face generated by $F(x)$ and $C_{P}$ is the same as the face generated by $F\left(\eta_{A, P}(x)\right)$ and $C_{P}$. To check this, let $x^{\prime}:=\eta_{A, P}(x)$, so that $\mu_{A}(x)=\mu_{A}\left(x^{\prime}\right)+p$ for some $p \in C_{P}$. This implies that $p$ and $\mu_{A}\left(x^{\prime}\right)$ belong to $F:=\left\langle\mu_{A}(x)\right\rangle$, and hence that $F$ is the face of $C_{Q}$ generated by $\mu_{A}\left(x^{\prime}\right)$ and $F \cap C_{P}$. Now recall that $F(x)$ is the face of $C_{Q}$ generated by $\mu_{A}(x)$ and similarly for $x^{\prime}$.

Proof of Theorem 2.5 Fix an element $q$ of $Q$ and let $\rho_{P, q}: C_{P} \rightarrow X_{P}$ be the composite

$$
\rho_{P, q}: C_{P} \stackrel{p \mapsto q+\theta(p)}{\longrightarrow} C_{Q} \stackrel{\nu_{A}}{\longrightarrow} X_{Q} \stackrel{X_{\theta}}{\longrightarrow} X_{P} .
$$

Let us note that if $F$ is a face of $P$, then $\theta$ induces a map $C_{F} \rightarrow C_{Q}$, and we also find a map $\rho_{F, q}: C_{F} \rightarrow X_{F}$. The following shows that, with suitable hypotheses, the maps $\rho_{P, q}$ and $\rho_{F, q}$ are compatible.

Lemma 2.7 Let $P$ and $Q$ be fine monoids and let $\theta: C_{P} \rightarrow C_{Q}$ be an injective homomorphism of their corresponding cones. Suppose that $q \in C_{Q}$ is contained in a face $G$ of $C_{Q}$ such that $G^{\mathrm{gp}} \cap P^{\mathrm{gp}}=0$ and such that the map $C_{P} \rightarrow C_{Q} / G$ is exact. Then the map $\rho_{P, q}: C_{P} \rightarrow X_{P}$ in (3) is compatible with the stratifications by faces: it sends each face $C_{F}$ of $C_{P}$ to the corresponding subset $X_{F}$ of $X_{P}$, and there is a commutative diagram

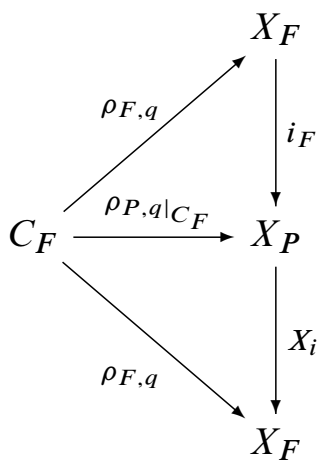

where $i: F \rightarrow P$ is the inclusion.

Proof The lower triangle of the diagram commutes by the definitions of $\rho_{P, q}$ and $\rho_{F, q}$, and the upper triangle will commute as soon as $\rho_{P, q}$ maps $C_{F}$ to $X_{F}$. Since $C_{P} \rightarrow C_{Q} / G$ is exact, each face $F$ of $C_{P}$ lifts to a face of $C_{Q} / G$. Thus there exists a face $G^{\prime}$ of $C_{Q}$ containing $G$ such that $G^{\prime} \cap C_{P}=F$. Choose a homomorphism 
$h: C_{Q} \rightarrow\left(\mathbf{R}_{\geq},+\right)$with $h^{-1}(0)=G^{\prime}$. If $p \in F$, then $q+p \in G^{\prime}$, and so if $x:=$ $v_{A}(q+p)$,

$$
0=h(q+p)=\sum_{s} a_{s} x(s) h(s)
$$

Since $a_{s}>0$ for all $s$ in the support of $A$ and $h(s) \neq 0$ if $s \notin G^{\prime}$, it follows that $x(s)=0$ if $s \notin G^{\prime}$. This means that $x \in X_{G^{\prime}} \subseteq X_{Q}$, and hence that $\rho_{P, q}(p):=$ $X_{\theta}(x) \in X_{F} \subseteq X_{P}$.

Before proceeding, let us review some elementary compatibilities about bilinear forms in our context. Let $V$ be a finite dimensional real vector space, let $V^{\vee}$ be its dual, and let $\tau: V^{\vee} \rightarrow V$ be a linear map. Recall that the associated bilinear form $\beta$ on $V^{\vee}$ is defined by

$$
\beta(\phi, \psi):=\psi(\tau(\phi)) .
$$

Symmetry of $\beta$ means that the diagram

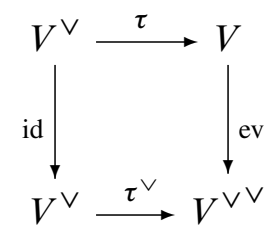

commutes. Assume now that $\beta$ is symmetric and positive definite. Then $\tau$ is an isomorphism and the bilinear form on $V$ corresponding to the map $\tau^{-1}: V \rightarrow V^{\vee}$ is just the bilinear form obtained from $\beta$ by transport of structure using the isomorphism $\tau$. Let us denote this bilinear form (which is also positive definite and symmetric) also by $\beta$. Note that if $W$ is a linear subspace of $V$, then $\beta$ restricts to a positive definite bilinear form on $W$ and hence defines an isomorphism $W \rightarrow W^{\vee}$. In fact the following diagram also commutes:

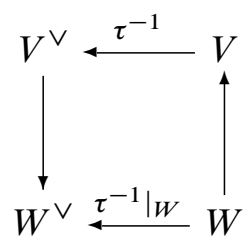

Let $F$ be a face of $P$. By Lemma 2.7, $\rho_{P, q}$ induces a map $C_{F} \rightarrow X_{F} \subseteq X_{P}$, which we can identify with $\rho_{F, q}$, and we just write $\rho_{q}$ in either case. Note that the map $+_{q}: p \mapsto q+\theta(p)$ maps $C_{F}$ into the face $C_{G}$ of $C_{Q}$ generated by $\theta(F)$ and $q$ and induces a map $C_{F}^{o} \rightarrow C_{G}^{o}$. To simplify the notation, we replace $Q$ by $G$. If $p \in F$, 
let $x:=\rho_{q}(p)$. Then we have commutative diagrams:
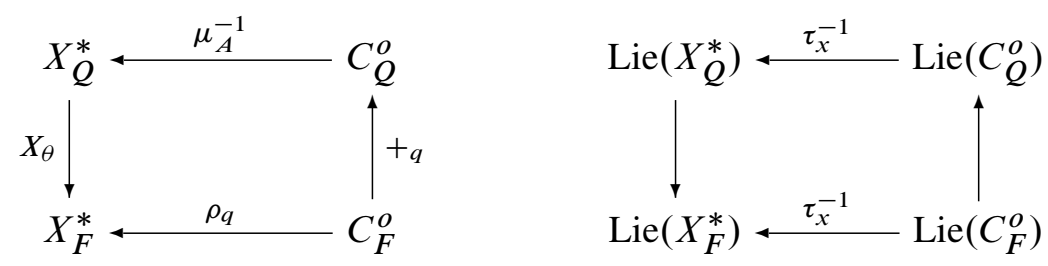

Here the diagram on the right is the derivative of the diagram on the left, and identifies with diagram (4). Thus $d \rho_{q}$ at $p$ can be identified with $\tau_{x}^{-1}$.

Lemma 2.8 Let $q \in C_{Q}$ be as in Lemma 2.7 and let $F$ be a face of $P$. The restriction of $\rho_{q}$ to the interior of $C_{F}^{o}$ is a homeomorphism onto its image, which is an open subset of $X_{F}^{*} \subseteq X_{P}$.

Proof It follows from diagrams (5) and (4) that if $p \in C_{F}^{o}$, the derivative of the map $\rho_{q}: C_{F} \rightarrow X_{F}$ at $p$ is the isomorphism $V_{F} \rightarrow V_{F}^{\vee}$ corresponding to the restriction of the bilinear form $\beta_{\rho_{q}(p)}$ on $V_{Q}$ to $V_{F}$. By the implicit function theorem, the restriction of $\rho_{q}$ to $C_{F}^{o}$ is an isomorphism locally on $C_{F}^{o}$, and in particular its image is an open subset of $X_{F}^{*}$.

Let $p$ and $p^{\prime}$ be two distinct elements of $C_{F}^{o}$, let $v:=p^{\prime}-p \in V_{F}$, and for $t \in[0,1]$ let $f(t):=p+t v$. Then $f$ is a continuous map from $[0,1]$ to $C_{F}^{o}$, and its derivative at any $t \in(0,1)$ is $v$. The logarithm map induces an isomorphism $X_{F}^{*} \cong \operatorname{Hom}\left(F^{\mathrm{gp}},(\mathbf{R},+)\right)$, and evaluation at $v$ defines a map $\log \left(e_{v}\right): X_{F}^{*} \rightarrow(\mathbf{R},+)$. Recall that $d \log \left(e_{v}\right)$ is the invariant differential form corresponding to the element $v \in V \cong \operatorname{Lie}\left(X_{F}^{*}\right)^{\vee}$, and that the derivative of the map $C_{F}^{o} \rightarrow X_{F}^{*}$ is the isomorphism corresponding to $\beta_{\rho_{q}}$. It follows that the derivative of the composite $\log \left(e_{v}\right) \circ \rho_{q} \circ f$ is $\beta_{\rho_{q} \circ f}(v, v)$. Since $\beta$ is everywhere positive definite, this function is increasing. Hence $\log \left(e_{v}\right)\left(\rho_{q}(p)\right) \neq$ $\log \left(e_{v}\right)\left(\rho_{q}\left(p^{\prime}\right)\right)$, so $\rho_{q}(p) \neq \rho_{q}\left(p^{\prime}\right)$, as required.

Lemma 2.9 Let $\left(q_{n}, p_{n}\right)$ be a sequence in $C_{Q} \times C_{P}$. If $\left(q_{n}\right)$ is bounded and $\left(p_{n}\right)$ is unbounded, then $\rho_{q_{n}}\left(p_{n}\right)$ is unbounded.

Proof Choose any norm on $V_{P}$, and let $\lambda_{n}:=\left\|p_{n}\right\|$. Replacing $\left(q_{n}, p_{n}\right)$ by a subsequence, we may assume that $\lambda_{n} \neq 0$ for all $n$ and that the sequence $\left(\lambda_{n}\right)$ tends to infinity. Let $p_{n}^{\prime}:=p_{n} / \lambda_{n}$. Then $p_{n}^{\prime}$ is a sequence in the unit ball of $V_{P}$, and hence contains a convergent subsequence. Passing to this subsequence, we may assume that $\left(p_{n}^{\prime}\right)$ converges to some $p^{\prime}$ in the unit ball. Let $x_{n}:=v_{A}\left(q_{n}+p_{n}\right)$. Let $S$ be the 
support of $A$, let $S_{u}$ be the set of all $s \in S$ such that $x_{n}(s)$ is unbounded and let $S_{b}$ be its complement. Then $\left(\lambda_{n}^{-1} q_{n}\right)$ approaches zero, and

$$
\lambda_{n}^{-1} q_{n}+p_{n}^{\prime}=\lambda_{n}^{-1} \mu_{A}\left(x_{n}\right)=\sum_{s \in S_{u}} \lambda_{n}^{-1} a_{s} x_{n}(s) s+\sum_{s \in S_{b}} \lambda_{n}^{-1} a_{s} x_{n}(s) s .
$$

The second sum on the right side converges to zero, and it follows that the first sum converges to $p^{\prime}$. Since $p^{\prime} \neq 0, S_{u}$ is not empty. Choose some $s_{1} \in S_{u}$, and let $\left(n_{k}\right)$ be an increasing sequence such that the subsequence $\left(x_{n_{k}}\left(s_{1}\right)\right)$ tends to infinity. Let $S_{u}^{\prime}$ be the set of all $s$ such that $x_{n_{k}}(s)$ is unbounded and let $S_{b}^{\prime}$ be its complement. Then equation (6) holds with the subsequence $\left(q_{n_{k}}, p_{n_{k}}\right)$ in place of $\left(q_{n}, p_{n}\right)$ and with $S_{u}$ and $S_{b}$ replaced by $S_{u}^{\prime}$ and $S_{b}^{\prime}$. Furthermore $s_{1} \in S_{u}^{\prime} \subseteq S_{u}$. After repeating this process a finite number of times, we find a subsequence of the original sequence with the property that $S=S_{u} \cup S_{b}$, where $\left(x_{n}(s)\right)$ approaches infinity if $s \in S_{u}$ and is bounded if $s \in S_{b}$. Equation (6) still holds, and hence $p^{\prime}$ is contained in the closure of the subcone $C_{S_{u}}$ of $C_{P}$ spanned by $S_{u}$. Since $S_{u}$ is finite, this cone is already closed, and so in fact $p^{\prime} \in C_{S_{u}}$. Write $p^{\prime}=\sum\left\{c_{s^{\prime}} s^{\prime}: s^{\prime} \in S_{u}\right\}$, where $c_{s^{\prime}} \geq 0$. Then $x_{n}\left(p^{\prime}\right)=\prod x_{n}\left(s^{\prime}\right)^{c_{s^{\prime}}}$. Since each sequence $\left(x_{n}\left(s^{\prime}\right)\right)$ tends to infinity and at least one $c_{S^{\prime}}$ is positive, $\left(x_{n}\left(p^{\prime}\right)\right)$ is unbounded. Since $p^{\prime} \in C_{P}$, it follows that $\left(X_{\theta}\left(x_{n}\right)\right)$ is unbounded in $X_{P}$.

We can now finish the proof of Theorem 2.5. From Theorem 1.4 and Corollary 2.4 we have homeomorphisms

$$
\mu_{A}: X_{Q} \rightarrow C_{Q} \quad \text { and } \quad g: C_{Q, P} \times C_{P} \rightarrow C_{Q}
$$

Thus it will suffice to show that $h:=\left(\eta_{A, P}, X_{\theta}\right) \circ \mu_{A}^{-1} \circ g$ is a homeomorphism. This is the map

$$
h: C_{Q, P} \times C_{P} \rightarrow X_{Q, P} \times X_{P}, \quad(q, p) \mapsto\left(\mu_{A}^{-1}(q), \rho_{q}(p)\right) .
$$

It is clear that $h$ is continuous, and we claim that it is also proper. To see this let $K$ be a compact subset of $X_{Q, P} \times X_{P}$, let $\left(q_{n}, p_{n}\right)$ be a sequence in $h^{-1}(K)$, and let $\left(x_{n}, y_{n}\right):=h\left(q_{n}, p_{n}\right)$. Since $q_{n}=\mu_{A}\left(x_{n}\right)$ and $\left(x_{n}\right)$ is contained in a compact set, $\left(q_{n}\right)$ is bounded. Since $\left(y_{n}\right)=\left(\rho_{q_{n}}\left(p_{n}\right)\right)$, it follows from Lemma 2.9 that $\left(p_{n}\right)$ is also bounded. Thus $h^{-1}(K)$ is closed and bounded, hence compact. Next we check that $h$ is bijective. Since $\mu_{A}$ is a bijection $X_{Q, P} \rightarrow C_{Q, P}$, it will suffice to prove that each $\rho_{q}$ is bijective. We know that $\rho_{q}$ is compatible with the stratifications of $C_{\boldsymbol{P}}$ and $X_{P}$ by faces, and from Lemma 2.8 that the restriction of $\rho_{q}$ to each $C_{F}^{o}$ is injective and has nonempty open image. Since the restriction of $\rho_{q}$ to $C_{F}^{o}=\rho_{q}^{-1}\left(X_{F}^{*}\right)$ is still proper, its image is also closed, and hence $\rho_{q}$ is surjective, as required. Thus $h$ is continuous, bijective, and proper, hence a homeomorphism. 
Remark 2.10 It is also possible to give a direct proof of the surjectivity of $h$, as we did for the moment map in Remark 1.6. It suffices to prove that for each $q \in C_{Q}$ as in Lemma 2.7, the map $\rho_{q}: C_{P} \rightarrow X_{P}$ is surjective. Let $F$ be a face of $P$. We shall prove that the map $\rho_{q}: C_{F}^{o} \rightarrow X_{F}^{*}$ is surjective. We may as well take $F=P$. Choose a generating effective cycle $B$ of $C_{P}$ and recall that the moment map $\mu_{B}: X_{P}^{*} \rightarrow C_{P}^{o}$ is injective. Thus it suffices to prove that for each $c \in C_{P}^{o}$, there is a point $p \in C_{P}$ such that $\mu_{B} \circ \rho_{q}(p)=c$. We follow the method of Remark 1.6. Consider the function

$$
\Psi_{c}=e_{c} \exp \left(-e_{B}\right): X_{P} \rightarrow \mathbf{R}_{\geq},
$$

where $e_{B}:=\sum b_{p} e_{p}$. This function is nonzero on $X_{P}^{*}$ and $d \log \Psi_{c}=c-\mu_{B}$ on $X_{P}^{*}$. Thus it suffices to show that there is some point $x$ in the image $U_{q}^{*}$ of the restriction of $\rho_{q}$ to $C_{P}^{o}$ at which $d \log \Psi_{c}=0$. We know already that $U_{q}^{*}$ is open in $X_{P}^{*}$, so it suffices to show that $\Psi_{c}$ has a critical point somewhere on $U_{q}^{*}$. Thus it will suffice to show that $\Psi_{c}$ has a maximum somewhere on $U_{q}^{*}$, or equivalently, that $\Psi_{c} \circ \rho_{q}$ has a maximum somewhere on $C_{P}^{o}$. For each $r>0$, let $C_{B, c}(r)$ denote the set of points of $C_{P}$ where $\Psi_{c} \circ \rho_{q}$ is at least $r$, and let $X_{B, c}(r)$ denote the set of points of $X_{P}$ where $\Psi_{c}$ is at least $r$. Note that since $\Psi_{c}$ vanishes on $X_{P} \backslash X_{P}^{*}, C_{B, c}(r)$ is contained in $C_{P}^{o}$. Lemma 1.7, applied to $P, B$, and $c$, implies that $X_{B, c}(r)$ is bounded. Since $\rho_{q}\left(C_{B, c}(r)\right) \subseteq X_{B, c}(r)$, Lemma 2.9 implies that $C_{B, c}(r)$ is also bounded, hence compact. Choose some $p_{0} \in C_{P}^{o}$, and let $r:=1 / 2 \Psi_{c}\left(\rho_{q}\left(p_{0}\right)\right)$. Then $\Psi_{c} \circ \rho_{q}$ has a maximum on $C_{B, c}(r)$, which will be a maximum of $\Psi_{c} \circ \rho_{q}$ on all of $C_{P}^{o}$, as required.

As an offshoot of our technique, we can force the following functoriality for moment maps:

Proposition 2.11 Let $Q$ be a fine monoid and let $\theta: P \rightarrow Q$ be the inclusion of an exact submonoid. Let $S$ (resp. $T$ ) be a finite set of generators for $Q$ (resp. $P$ ). Then

$$
X_{\theta} \circ \mu_{S}^{-1} \circ C_{\theta}: C_{P} \rightarrow X_{P}
$$

is a homeomorphism which sends each face $C_{F}$ of $C_{P}$ to the corresponding subset $X_{F}$ of $X_{P}$. Hence there is a stratification preserving homeomorphism $h_{S, T}: X_{P} \rightarrow X_{P}$ which makes the following diagram commute:

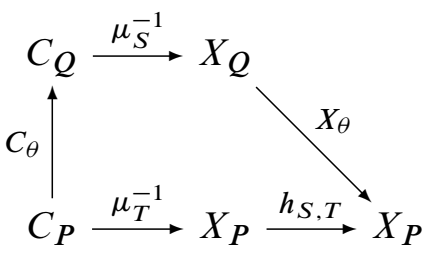


Proof The proof of Theorem 2.5 shows that $\rho_{0}$ is a homeomorphism when $\theta: P \rightarrow Q$ is the inclusion of an exact submonoid. Then $h_{S, T}:=\rho_{0} \circ \mu_{T}$ fits into the diagram shown.

Proof of Theorem 0.2 The only difficulty is that in Theorem 0.2 , the monoid $P$ need not be sharp. To reduce to Theorem 2.5 , let $\bar{Q}:=Q / Q^{*}$ and $\bar{P}:=P / P^{*}$ and consider the diagram of finite dimensional vector spaces:

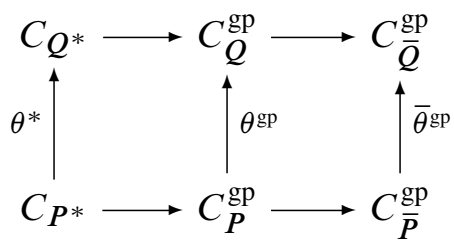

Note that since $\theta$ is local, the map $\bar{\theta}^{\mathrm{gp}}$ is injective. Choose a splitting $C_{Q}^{\mathrm{gp}} \rightarrow C_{Q^{*}}$ which maps $C_{P}^{\mathrm{gp}}$ to $C_{P^{*}}$. Note that the corresponding splittings $C_{\bar{Q}}^{\mathrm{gp}} \rightarrow C_{Q}^{\mathrm{gp}}$ and $C_{\bar{P}}^{\mathrm{gp}} \rightarrow C_{P}^{\mathrm{gp}}$ necessarily map $C_{\bar{Q}}$ to $C_{Q}$ and $C_{\bar{P}}$ to $C_{P}$, respectively. It follows that there is a commutative diagram:

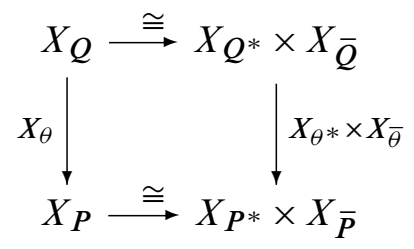

Since $C_{P^{*}} \rightarrow C_{Q^{*}}$ is an injective map of vector spaces, it admits a splitting, and hence the map $X_{\theta^{*}}$ is isomorphic to a projection map. Thus $X_{\theta}$ will be isomorphic to a projection if $X_{\bar{\theta}}$ is. Since $\theta$ is local, the map $\bar{\theta}^{\text {gp }}$ is injective. Then $\bar{\theta}$ is a locally exact and injective map of sharp cones, and it suffices to apply Theorem 2.5 to $\bar{\theta}$.

Theorem 2.5 implies that (with the hypothesis there) all the fibers of the map $X_{Q} \rightarrow X_{P}$ are homeomorphic. For each point $y$ of $X_{P}$, let $X_{Q}(y)$ denote the fiber of $X_{\theta}$ over $y$. In particular, the "broken" fiber $X_{Q}(v)=X_{Q, P}$ over the vertex $v$ of $X_{P}$ is homeomorphic to the fiber $X_{Q}(1)$ over 1 , where 1 is the identity element of the monoid $X_{P}$. Explicitly, if $y \in X_{Q}(v)$, there is a unique $s(y) \in X_{Q}(1)$ such that $\eta_{A, P}(s(y))=y$, and $s: X_{Q}(v)=X_{Q, P} \rightarrow X_{Q}(1)$ is a homeomorphism. Let

$$
\eta_{A, P}^{\prime}:=s \circ \eta_{A, P}: X_{Q} \rightarrow X_{Q}(1) .
$$

We obtain the following reformulation of Theorem 2.5, which is sometimes more convenient. 
Corollary 2.12 With the hypotheses of Theorem 2.5, there is a continuous map $\eta_{A, P}^{\prime}: X_{Q} \rightarrow X_{Q}(1)$ with the following properties:

(1) The map $\left(\eta_{A, P}^{\prime}, X_{\theta}\right): X_{Q} \rightarrow X_{Q}(1) \times X_{P}$ is a homeomorphism, and the diagram

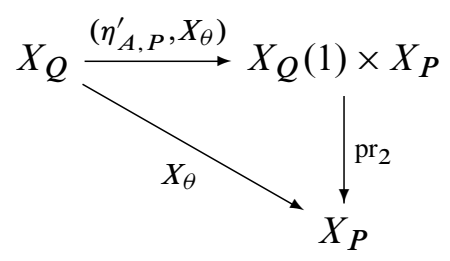

is commutative.

(2) For each $x \in X_{Q}, F\left(\eta_{A, P}^{\prime}(x)\right)$ is the face of $Q$ generated by $F(x)$ and $P$.

Proof Let $x$ be a point in $X_{Q}$, and let $y:=\eta_{A, P}(x)$ and $y^{\prime}:=\eta_{A, P}^{\prime}(x)=s(y)$. Then $\eta_{A, P}\left(y^{\prime}\right)=y$, and it follows from Remark 2.6 that

$$
\langle F(x)+P\rangle=\langle F(y)+P\rangle=\left\langle F\left(y^{\prime}\right)+P\right\rangle .
$$

Since $y^{\prime} \in X_{Q}(1), F\left(y^{\prime}\right)$ already contains $P$, so $F\left(\eta_{A, P}^{\prime}(x)\right)$ is the face generated by $F(x)$ and $P$.

Remark 2.13 Let us attempt to describe the fibers of the map $X_{\theta}$ in Corollary 2.12. The fiber $X_{Q}(1)$ consists of the set of homomorphisms $x: C_{Q} \rightarrow\left(\mathbf{R}_{\geq}, \cdot\right)$ sending $C_{P}$ to 1 , or, equivalently, the set of homomorphisms $C_{Q} / C_{P} \rightarrow\left(\mathbf{R}_{\geq}, \cdot\right)$, where $C_{Q} / C_{P}$ is the image of $C_{Q}$ in the quotient $C_{Q}^{\mathrm{gp}} / C_{P}^{\mathrm{gp}}$. Note that this identification $X_{Q}(1) \cong$ $X_{C_{Q} / C_{P}}$ is compatible with the stratification by faces: if $x \in X_{Q}(1)$ corresponds to $x^{\prime} \in X_{C_{Q} / C_{P}}$, then $F(x)$ is the face of $C_{Q}$ containing $C_{P}$ corresponding naturally to the face $F\left(x^{\prime}\right)$ of $C_{Q} / C_{P}$. We will allow ourselves to identify the faces of $C_{Q} / C_{P}$ with those faces of $C_{Q}$ containing $C_{P}$ when convenient.

A morphism of monoids or cones $\theta: P \rightarrow Q$ is said to be dominating or vertical if the image $Q / P$ of $Q$ in $\operatorname{Cok}\left(\theta^{\mathrm{gp}}\right)$ is a group, or equivalently if the image of $P$ is not contained in any proper face of $Q$. If $\theta: C_{P} \rightarrow C_{Q}$ is vertical, then $X_{Q}(1) \subseteq X_{Q}^{*}$ and in fact $X_{Q}(1)$ is a smooth submanifold of $X_{Q}^{*}$. Indeed, $X_{Q}(1) \cong X_{C_{Q} / C_{P}}$ and $C_{Q} / C_{P}$ is a group, so that in fact $X_{C_{Q} / C_{P}}$ is homeomorphic to a Euclidean space. More specifically, if $x \in X_{Q}$ we say that $X_{\theta}$ is vertical at $x$ if the localization of $Q$ by $F(x)+P$ is a group, or equivalently, if $\langle F(x)+P\rangle=Q$. It is clear that the set $X_{Q}^{v}$ of all $x$ at which $X_{\theta}$ is vertical is an open subset of $X_{Q}$.

The following result describes the fibers of the submersions arising in Theorem 2.5. 
Proposition 2.14 Let $Q$ and $P$ be fine monoids.

(1) There exist a manifold with boundary $(M, B)$ and homeomorphisms

$$
(M, M \backslash B) \cong\left(X_{Q}, X_{Q}^{*}\right) \cong\left(C_{Q}, C_{Q}^{o}\right) .
$$

In particular, the boundary $B$ is empty if and only if $Q$ is a group.

(2) Let $\theta: C_{P} \rightarrow C_{Q}$ be an exact, injective, and locally exact morphism of fine monoids, and let $X_{Q}^{v} \subseteq X_{Q}$ denote the open subset of points at which $X_{\theta}$ is vertical. Then there is a commutative diagram

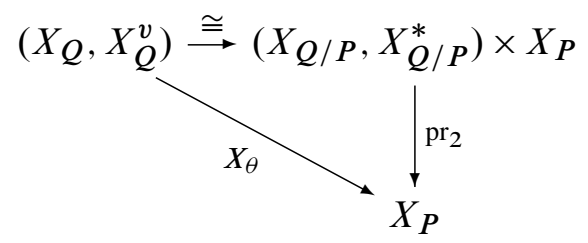

in which the top horizontal arrow is a homeomorphism. In particular, the fibers of $X_{Q} \rightarrow X_{P}$ are all topological manifolds with boundary, and the boundary is empty if and only if $\theta$ is vertical.

Proof Although statement (1) is well-known, we include a proof for the sake of completeness. The moment map associated with any effective generating cycle induces a homeomorphism

$$
\left(X_{Q}, X_{Q}^{*}\right) \cong\left(C_{Q}, C_{Q}^{o}\right)
$$

so it suffices to prove that $\left(C_{Q}, C_{Q}^{o}\right)$ is homeomorphic to some $(M, M \backslash B)$. Choose a splitting $C_{Q} \cong C_{Q^{*}} \times C_{\bar{Q}}$. Then

$$
\left(C_{Q}, C_{Q}^{o}\right) \cong\left(C_{Q^{*}} \times C_{\bar{Q}}, C_{Q^{*}} \times C_{\bar{Q}}^{o}\right),
$$

and $C_{Q^{*}}$ is a Euclidean space. Hence it suffices to prove the result when $Q$ is sharp. Choose an element $p$ of the interior of $Q$ and let $P$ be the submonoid of $Q$ generated by $p$. Then $P \rightarrow Q$ is exact and locally exact, and the quotient $Q / P$ is a group. It follows from Corollary 2.4 that the projection mapping $C_{Q} \rightarrow C_{Q / P}$ induces a homeomorphism $C_{Q, P} \rightarrow C_{Q / P}$, and then a homeomorphism $C_{Q / P} \times C_{P} \rightarrow C_{Q}$, sending $C_{Q / P} \times C_{P}^{o}$ to $C_{Q}^{o}$. Alternatively one can argue from Corollary 2.12. As we saw in Remark 2.13, $X_{Q}(1) \cong X_{Q / P}$ is homeomorphic to a Euclidean space $\mathbf{R}^{n-1}$, where $n$ is the dimension of $C_{Q}$. Thus $X_{Q}(1) \times \mathbf{R}_{\geq}$is a manifold with boundary $X_{Q}(1) \times\{0\}$. Since $X_{\theta}^{-1}\left(\mathbf{R}_{>}\right)=X_{Q}^{*}$, the homeomorphism of Corollary 2.12 produces the desired homeomorphism

$$
\left(X_{Q}, X_{Q}^{*}\right) \cong\left(X_{Q}(1) \times \mathbf{R}_{\geq}, X_{Q}(1) \times \mathbf{R}_{>}\right) .
$$


The horizontal arrow in the diagram of statement (2) is the same as that of the diagram in statement (1) of Corollary 2.12. Statement (2) of this corollary implies that this arrow maps $X_{Q}^{v}$ to $X_{Q / P}^{*}$. Statement (1) of the proposition, applied to the fine monoid $Q / P$, tells us that $\left(X_{Q / P}, X_{Q / P}^{*}\right)$ is a topological manifold with boundary.

\section{Submersivity of $\log$ smooth maps}

The main result of this section is Theorem 3.5 below. Before stating it, we shall review the notion of a $\log$ analytic space $X$ and its associated topological space $X_{\log }$. Let us begin with a version for toric varieties, which serve as local models for smooth log analytic spaces.

Let $Q$ be a fine monoid and let $A_{Q}$ denote the set of homomorphisms $Q \rightarrow(\mathbf{C}, \cdot)$, ie, the set of complex points of $\operatorname{Spec} \mathbf{C}[Q]$. We endow $A_{Q}$ with its natural structure of a complex analytic variety, which is the analytic space associated to an affine toric variety. ${ }^{2}$ As alluded to in the introduction, there is a natural way to introduce polar coordinates for such varieties. Recall that $X_{Q}:=\operatorname{Hom}\left(Q,\left(\mathbf{R}_{\geq}, \cdot\right)\right)$, which is naturally a subset of $A_{Q}$. Similarly we define $T_{Q}:=\operatorname{Hom}\left(Q, \mathbf{S}^{1}\right) \subseteq A_{Q}$, where $\mathbf{S}^{1}:=\{z \in \mathbf{C}:|z|=1\}$. Then $T_{Q}$ acts naturally on $A_{Q}$ by multiplication. If $x \in X_{Q}$, the isotropy subgroup of $T_{Q}$ at $x$ is $T_{Q / F(x)} \subseteq T_{Q}$. Furthermore, there is a natural retraction $A_{Q} \rightarrow X_{Q}$, sending a point $z$ to the point $|z|$. The following proposition is then immediate.

\section{Proposition 3.1 Let}

$$
\tau_{Q}: A_{Q}^{\log }:=T_{Q} \times X_{Q} \rightarrow A_{Q}
$$

be the map induced by the action of $T_{Q}$ on $A_{Q}$ and the inclusion $X_{Q} \rightarrow A_{Q}$. Then $\tau_{Q}$ is surjective and proper, and for $z \in A_{Q}, \tau_{Q}^{-1}(z) \cong T_{Q / F(|z|)}$.

We can now state the following local version of Theorem 3.5.

Proposition 3.2 Let $\theta: P \rightarrow Q$ be a locally exact and injective homomorphism of fine monoids. Then the map $A_{\theta}^{\log :} A_{Q}^{\log } \rightarrow A_{P}^{\log }$ is submersive. Its fibers are topological manifolds with boundary, and the boundary consists of those points of $A_{Q}^{\log }$ lying over points of $X_{Q}$ at which $X_{\theta}$ is not vertical (see Remark 2.13).

Proof Replacing a monoid $P$ by its saturation does not change $A_{P}^{\log }$, so we may and shall assume that $P$ and $Q$ are saturated. Let $F:=\theta^{-1}\left(Q^{*}\right)$. Then $F$ is a face

\footnotetext{
${ }^{2}$ Strictly speaking $A_{Q}$ is traditionally called a toric variety only when $Q$ is fine and saturated and $Q^{\mathrm{gp}}$ is torsion free.
} 
of $P$ and $\theta$ factors: $\theta=\theta^{\prime} \circ \theta^{\prime \prime}$, where $\theta^{\prime \prime}: P \rightarrow P_{F}$ and $\theta^{\prime}: P_{F} \rightarrow Q$. Since the composition of two submersive maps is submersive, it suffices to prove the result for $\theta^{\prime}$ and $\theta^{\prime \prime}$. The map $A_{\theta^{\prime \prime}}: A_{P_{F}} \rightarrow A_{P}$ is an open immersion, and the same is true for $A_{\theta^{\prime \prime}}^{\log }$, and hence it is certainly submersive. The map $\theta^{\prime}: P_{F} \rightarrow Q$ is local and locally exact, hence exact. Thus we are reduced to proving the proposition when $\theta$ is exact and locally exact. Since $A_{\theta}^{\log } \cong T_{\theta} \times X_{\theta}$ and the product of two submersions is a submersion, it will suffice to prove that $T_{\theta}$ and $X_{\theta}$ are submersive. Theorem 0.2 says that $X_{\theta}$ is a projection mapping, hence submersive.

The proposition will now follow if we prove that $T_{\theta}$ is submersive. This is almost immediate. Let $P^{\prime}$ (resp. $Q^{\prime}$ ) be the quotient of $P$ (resp. $Q$ ) by the torsion subgroup $P_{t}$ of $P^{\mathrm{gp}}$ (which is contained in $P$ because $P$ is saturated). Then the natural map $T_{P} \rightarrow T_{P_{t}}\left(\right.$ resp. $\left.T_{Q} \rightarrow T_{P_{t}}\right)$ makes $T_{P}\left(\right.$ resp. $\left.T_{Q}\right)$ a torsor over $T_{P^{\prime}}\left(\right.$ resp. $T_{Q^{\prime}}$ ). Thus we are reduced to the case in which $P^{\mathrm{gp}}$ is torsion free. Consider the exact sequence of finitely generated abelian groups

$$
0 \rightarrow P^{\mathrm{gp}} \rightarrow Q^{\mathrm{gp}} \rightarrow G \rightarrow 0
$$

where $G$ is by definition the quotient of $Q^{\text {gp }}$ by the image of $P^{\text {gp }}$. The obstruction $\xi$ to splitting this sequence lies in $\operatorname{Ext}^{1}\left(G, P^{\mathrm{gp}}\right)$, which is a finite group. It follows that there is natural number $n$ such that $n \xi=0$. This means that the sequence obtained by pushout

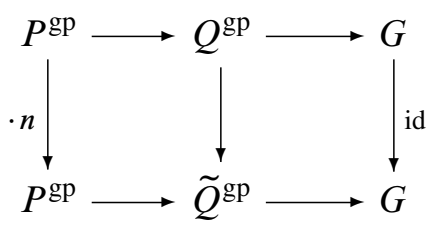

splits, and hence that the corresponding map $T_{\widetilde{\theta}}: T_{\widetilde{Q}^{\mathrm{gp}}} \rightarrow T_{P^{\mathrm{gp}}}$ is a product map. On the other hand, the square in the diagram

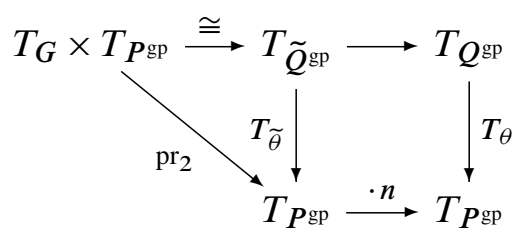

is Cartesian, and the bottom arrow is a covering space. It follows that $T_{\theta}$ is a fiber bundle, and in particular is submersive.

Finally, let us remark that the fiber of $A_{\theta}^{\log }$ over a point $(\zeta, x)$ of $A_{P}^{\log }$ is just the product of the fiber of $T_{\theta}$ over $\zeta$ and the fiber of $X_{\theta}$ over $x$. The former is the manifold $T_{G}$ and the latter is a manifold with boundary as described in Proposition 2.14. This proves the last statement. 
Remark 3.3 The proof shows that if $P \rightarrow Q$ is local as well as locally exact then $A_{\theta}^{\log }$ is a fiber bundle, and if in addition the cokernel of $P^{\mathrm{gp}} \rightarrow Q^{\mathrm{gp}}$ is torsion free, then in fact $A_{\theta}$ is a trivial fiber bundle, ie, is isomorphic to a projection mapping.

Let us briefly recall the basic definitions in log geometry. For details we refer to Kato [11] and Illusie, Kato and Nakayama [7].

Definition 3.4 A log structure on an analytic space $X$ is a morphism of sheaves of monoids

$$
\alpha: M_{X} \rightarrow\left(\mathcal{O}_{X}, \cdot\right)
$$

such that the induced map

$$
\alpha^{-1}\left(\mathcal{O}_{X}^{*}\right) \rightarrow \mathcal{O}_{X}^{*}
$$

is an isomorphism.

A $\log$ analytic space is an analytic space endowed with a $\log$ structure $\alpha$, and a morphism of log analytic spaces is a morphism which is compatible with the log structures in the evident sense. A basic example is the following. Let $Q$ be a monoid and let $\beta: Q \rightarrow\left(\mathcal{O}_{X}, \cdot\right)$ be a morphism of sheaves of monoids. Then one can form the pushout in the category of sheaves of monoids:

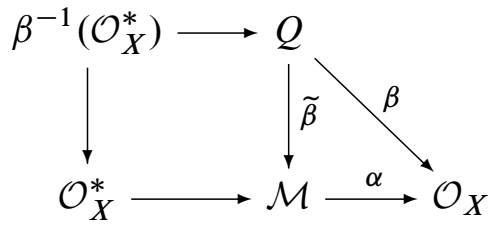

Then $\alpha$ is a $\log$ structure on $X$, and $\widetilde{\beta}$ is a chart for $\alpha$. A log structure or space is said to be coherent (resp. fine) if locally on $X$ it admits charts given by finitely generated (resp. fine) monoids. In particular, on the analytic space $A_{Q}$ associated to the toric variety $\operatorname{Spec} \mathbf{C}[Q]$ as above, there is a natural map $Q \rightarrow \mathcal{O}_{A_{Q}}$, which defines a fine $\log$ structure $\mathcal{M}_{Q} \rightarrow \mathcal{O}_{A_{Q}}$ on $A_{Q}$. When $Q$ is saturated, Kato's Theorem [12, 11.6] implies that $\mathcal{M}_{Q}$ can be identified with the sheaf of holomorphic functions on $A_{Q}$ which become invertible when restricted to $A_{Q}^{*}:=A_{Q^{\mathrm{gp}} \text {. }}$.

A morphism $f: X \rightarrow Y$ of integral $\log$ analytic spaces is said to be exact (resp. vertical) at $x \in X$, if the map of monoids

$$
f^{b}: M_{Y, f(x)} \rightarrow M_{X, x}
$$

is exact (resp. vertical) (see Definition 2.1 and Remark 2.13). We say simply that $f$ is exact (resp. vertical) if it is so at every $x$. Similarly, $f$ is said to be strict if for every $x \in X$, the induced map $\bar{M}_{Y, f(x)} \rightarrow \bar{M}_{X, x}$ is an isomorphism. For the definition of 
a smooth morphism of log spaces, we must refer to Kato's original article [11]. See Illusie, Kato and Nakayama [7, 2.2].

Associated to a log analytic space $(X, \alpha)$ is continuous map of topological spaces

$$
\tau_{X}: X_{\log } \rightarrow X
$$

which globalizes the polar coordinate construction for toric varieties described above. We present here a slight generalization of the original definition of Kato and Nakayama in [13]; note that our definition does not require the log structure to be coherent. By definition $X_{\log }$ is the set of pairs $(x, \sigma)$, where $x$ is a point of $X$ and $\sigma$ is a homomorphism of monoids fitting into the commutative diagram

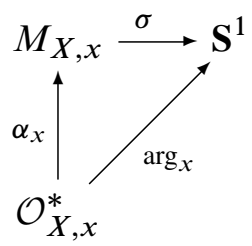

where $\arg _{x}(u):=u(x) /|u(x)|$. The map $\tau_{X}: X_{\log } \rightarrow X$ sends $(x, \sigma)$ to $x$. Corresponding to each section $m$ of $M_{X}$ on an open subset $U$ of $X$ is a function

$$
\arg (m): \tau_{X}^{-1}(U) \rightarrow \mathbf{S}^{1}, \quad(x, \sigma) \mapsto \sigma\left(m_{x}\right) .
$$

We endow $X_{\log }$ with the weak topology defined by the functions $\tau_{X}$ and $\arg (m)$ as $m$ ranges over the local sections of $M_{X}$. It follows from the fact that $\mathbf{S}^{1}$ is a compact and divisible group that the map $\tau_{X}$ is surjective and proper. The fiber of a point $x$ is a torsor under the group $\operatorname{Hom}\left(\bar{M}_{X, x}, \mathbf{S}^{1}\right)$.

Theorem 3.5 Let $f: X \rightarrow Y$ be an exact smooth morphism of fine log analytic spaces. Then $f_{\log }: X_{\log } \rightarrow Y_{\log }$ is a topological submersion. The fibers are topological manifolds with boundary, and the boundary consists of the set of points of $X_{\log }$ lying over points of $X$ at which $f$ is not vertical.

Proof We make use of the following argument from [7, A.3.3] to reduce to Proposition 3.2. The statement is local on $X$. Choose a point $x$ of $X$ and let $y:=f(x)$. It follows from the smoothness of $f$ that, after replacing $X$ by a neighborhood of $x$ and $Y$ by a neighborhood of $y$, there exist charts $P \rightarrow M_{Y}$ and $Q \rightarrow M_{X}$ and a morphism $\theta: P \rightarrow Q$ with the following properties. 
(1) The monoids $P$ and $Q$ are fine, and $\theta: P \rightarrow Q$ is injective.

(2) The maps $P \rightarrow \bar{M}_{Y, y}$ and $\bar{Q}:=Q / Q^{*} \rightarrow \bar{M}_{X, x}$ are isomorphisms.

(3) The diagram

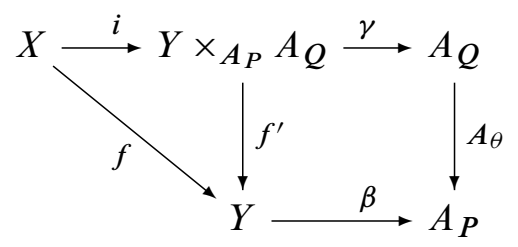

is commutative, its square is Cartesian, and $i$ is a strict open immersion.

Since $P$ is sharp, the vertex $v_{P}$ of $A_{P}$ is the point defined by the maximal ideal $P^{+}$ of $P$, and $\beta(y)=v_{P}$. Similarly, $\gamma(i(x))$ lies in the closed subset of $A_{Q}$ defined by the ideal $Q^{+}$of $Q$. Let $G$ be any face of $Q$ such that $\theta^{-1}(G)=0$ and let $\mathfrak{q}:=Q \backslash G$ be the complementary prime ideal. Then $A_{G} \cong Z(\mathfrak{q}) \subseteq A_{\theta}^{-1}\left(v_{P}\right)$, and $A_{G}^{*}$ is dense in $A_{G}$. Let $X_{y}:=f^{-1}(y)$, and note that since $i(x) \in \gamma^{-1}\left(A_{G}\right) \cap i\left(X_{y}\right)$, the latter is a nonempty open subset of $\gamma^{-1}\left(A_{G}\right)$ and hence meets $\gamma^{-1}\left(A_{G}^{*}\right)$. It follows that there is a point $x^{\prime}$ in $X_{y}$ which maps to some point of $A_{G}^{*}$. Since $Q \rightarrow M_{X}$ is a chart, the natural map $Q \rightarrow M_{X, x^{\prime}}$ induces an isomorphism $Q / G \rightarrow \bar{M}_{X, x^{\prime}}$. Since $f$ is exact, the map $M_{Y, y} \rightarrow M_{X, x^{\prime}}$ is exact, and it follows that $P \rightarrow Q / G$ is exact. Since this was true for an arbitrary face $G$ of $Q$ lying over the trivial face of $P$, it follows that $\theta$ is very locally exact (see Definition 2.1). By Theorem 2.3, $\theta$ is in fact exact and locally exact. By Proposition 3.2, $A_{\theta}^{\log }$ is submersive, and hence so is the base changed map $f_{\log }^{\prime}$. Since $i_{\log }$ is an open immersion, $f_{\log }$ is also submersive. The statement about the fibers also follows from Proposition 3.2, and this completes the proof.

Sometimes it is convenient to consider log structures which are not coherent (see for example Ogus [14; 15] and Gross and Siebert [4]). For example, if $M_{X} \rightarrow \mathcal{O}_{X}$ is a coherent $\log$ structure on $X$, any sheaf of faces $\mathcal{F}$ of $M_{X}$ is again a log structure, and it is often productive to work with $\mathcal{F} \rightarrow \mathcal{O}_{X}$ even if $\mathcal{F}$ is not coherent. When necessary to clarify with which $\log$ structure we are working, we will write $(X, \mathcal{F})$ or $X(\mathcal{F})$ to denote the $\log$ space $\left(X, \mathcal{F} \rightarrow \mathcal{O}_{X}\right)$, and similarly for $\mathcal{M}$.

The following definitions should be regarded as provisional.

Definition 3.6 Let $\mathcal{F} \rightarrow \mathcal{O}_{X}$ be a $\log$ structure on a complex analytic space $X$.

(1) $\mathcal{F}$ is relatively coherent if locally on $X$ there exists a coherent log structure $\mathcal{M}$ containing $\mathcal{F}$ such that $\mathcal{F}$ is locally generated as a sheaf of faces in $\mathcal{M}$ by a finite number of sections of $\mathcal{M}$. (In this case one says that $\mathcal{F}$ is relatively coherent in M.) 
(2) Let $Y$ be a fine log analytic space and let $f: X(\mathcal{F}) \rightarrow Y$ be a morphism of log analytic spaces. One says that $f$ is relatively smooth if locally on $X$ there exists a fine $\log$ structure $\mathcal{M}$ on $X$ in which $\mathcal{F}$ is relatively coherent satisfying the following conditions.

(a) The composed map $X(\mathcal{M}) \rightarrow X(\mathcal{F}) \rightarrow Y$ is smooth.

(b) The stalks of the quotient monoid $\mathcal{M} / \mathcal{F}$ are free monoids.

Let us remark that in the next theorem, which is so far our main justification for the above definition, the proof of submersivity will not use the condition (b). Our proof will show that, assuming only that (a) holds, then $f_{\log }(\mathcal{F})$ is a submersion whose fibers are locally the product of a manifold with boundary and a space with "toric singularities"; ie, a space homeomorphic to $A_{M}$ for some fine monoid $M$. Condition (b) will insure that this space is also a manifold with boundary.

Theorem 3.7 Let $Y$ be a fine $\log$ analytic space, let $\mathcal{F} \rightarrow \mathcal{O}_{X}$ be a relatively coherent $\log$ structure on an analytic space $X$, and let

$$
f(\mathcal{F}): X(\mathcal{F}) \rightarrow Y
$$

be an exact and relatively smooth morphism of log analytic spaces. Then the map $f_{\log }(\mathcal{F}): X_{\log }(\mathcal{F}) \rightarrow Y_{\log }$ is submersive. Furthermore, the fibers of $f_{\log }(\mathcal{F})$ are manifolds with boundary, and the boundary consists of those points lying over points of $X$ at which $f$ is not vertical.

Proof Since the statement is local on $X$ and $Y$, we may assume that there exists a fine $\log$ structure $\mathcal{M}$ on $X$ in which $\mathcal{F}$ is relatively coherent and such that $X(\mathcal{M}) \rightarrow Y$ is smooth. For each $x \in X, \mathcal{F}_{X}$ is a face of $\mathcal{M}_{X}$ and hence is an exact submonoid. By hypothesis, $\mathcal{M}_{Y, f(x)} \rightarrow \mathcal{F}_{x}$ is exact, and since the composite of two exact maps is exact, it follows that $\mathcal{M}_{Y, f(x)} \rightarrow \mathcal{M}_{x}$ is exact. Thus the map of log analytic spaces $X(\mathcal{M}) \rightarrow Y$ is smooth and exact.

Since $\mathcal{F}$ is relatively coherent in $\mathcal{M}$, we may assume that $\mathcal{F}$ is generated by a finite number of global sections $f_{i}$ of $\mathcal{M}$. Furthermore we may assume that there exist charts $P \rightarrow \mathcal{M}_{Y}$ and $Q \rightarrow \mathcal{M}$ and a morphism $\theta$ as in the proof of Theorem 3.5. We may also assume that each $f_{i}$ lifts to some $q_{i} \in Q$. Letting $G$ be the face of $Q$ generated by these $q_{i}$, we see that $\mathcal{F}$ is the sheaf of faces of $\mathcal{M}$ generated by the image of $G \rightarrow \mathcal{M}$. Let $A_{Q}\left(\mathcal{M}_{Q}\right)$ (resp. $A_{Q}(\mathcal{F})$ ) denote $A_{Q}$ with the log structure coming from $Q \rightarrow \mathcal{O}_{A_{Q}}$ (resp. from the sheaf of faces of $\mathcal{M}_{Q}$ generated by $G$ ). Then 
we have the following commutative diagram:

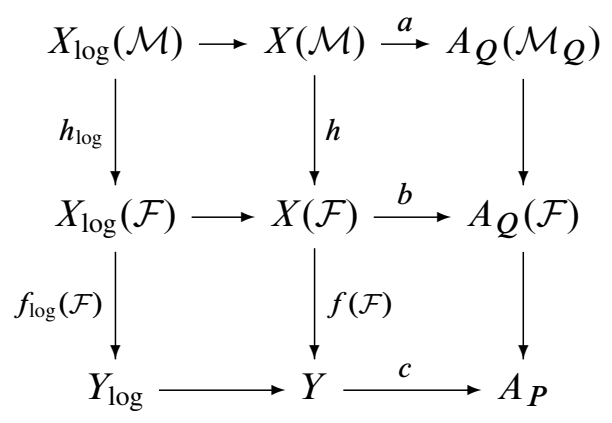

Here the map $a$ factors through a strict open immersion into the fiber product $Y \times_{A_{P}}$ $A_{Q}\left(\mathcal{M}_{Q}\right)$, and the map $h$ is an isomorphism on underlying analytic spaces. It follows that $b$ also factors through a strict open immersion into $Y \times_{A_{P}} A_{Q}(\mathcal{F})$. Then the top arrow in the diagram

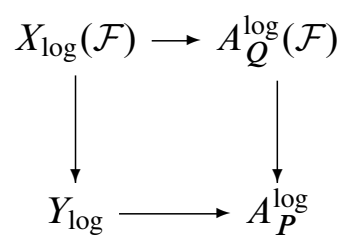

factors through an open immersion $X_{\log }(\mathcal{F}) \rightarrow Y_{\log } \times A_{P}^{\log } A_{Q}^{\log }(\mathcal{F})$. (Here we use a superscript "log" instead of a subscript for typographical reasons.) Thus we are reduced to the case when $X=A_{Q}, Y=A_{P}$, and $f \circ h$ is induced by a map $P \rightarrow G \rightarrow Q$. As in the proof of Theorem 3.5, it follows from the fact that $(X, \mathcal{M}) \rightarrow\left(Y, \mathcal{M}_{Y}\right)$ is smooth and exact that the map $P \rightarrow Q$ is locally exact. Since the statement is also local on $Y_{\log }$, we may replace $P \rightarrow Q$ by the pushout construction as in the proof of Proposition 3.2. Thus we may assume that the map $Q^{\mathrm{gp}} \rightarrow Q^{\mathrm{gp}} / P^{\mathrm{gp}}$ admits a splitting $\sigma$, which then induces an isomorphism

$$
T_{\sigma} \times T_{\theta}: T_{Q} \rightarrow T_{Q / P} \times T_{P}
$$

We have a factorization

$$
\tau_{\mathcal{M}}: T_{Q} \times X_{Q} \cong X_{\log }(\mathcal{M}) \stackrel{h_{\log }}{\longrightarrow} X_{\log }(\mathcal{F}) \stackrel{\tau_{\mathcal{F}}}{\longrightarrow} X
$$

in which all maps are proper and surjective. It follows that $X_{\log }(\mathcal{F})$ has the quotient topology induced by $h_{\log }$. If $z \in X, \tau_{\mathcal{M}}^{-1}(z)$ is a torsor under $T_{\overline{\mathcal{M}}_{z}}$ and $\tau_{\mathcal{F}}^{-1}(z)$ is a torsor under $T_{\overline{\mathcal{F}}_{z}}$. Hence if $z^{\prime} \in \tau_{\mathcal{F}}^{-1}(z), h_{\log }^{-1}\left(z^{\prime}\right)$ is a torsor under $T_{\overline{\mathcal{M}}_{z} / \overline{\mathcal{F}}_{z}}$. Let us identify $X_{\log }(\mathcal{M})$ with $A_{Q}^{\log }=T_{Q} \times X_{Q}$. Then if $z^{\prime \prime} \in \tau_{\mathcal{M}}^{-1}(z)$ corresponds to $(\zeta, x) \in T_{Q} \times X_{Q}, x=|z|$ and $F(x)$ is identified with the set of all $q \in Q$ which map to a unit of $\mathcal{M}_{z}$. Let $G(x)$ be the face of $Q$ generated by $F(x)$ and $G$. Then 
$\overline{\mathcal{M}}_{z} / \overline{\mathcal{F}}_{z} \cong Q / G(x)$, and the map $T_{Q} \times X_{Q} \rightarrow X_{\log }(\mathcal{F})$ identifies a pair of points $(\zeta, x)$ and $\left(\zeta^{\prime}, x^{\prime}\right)$ if and only if $x=x^{\prime}$ and $\zeta^{\prime} \zeta^{-1} \in T_{Q / G(x)} \subseteq T_{Q}$. Since $G(x)$ contains $P$, $T_{Q / G(x)}$ can be viewed as a subgroup of $T_{Q / P}$, and $\zeta^{\prime} \zeta^{-1}$ lies in $T_{Q / G(x)}$ if and only if $T_{\sigma}\left(\zeta^{\prime} \zeta^{-1}\right)$ lies in $T_{Q / G(x)}$ and $T_{\theta}\left(\zeta^{\prime} \zeta^{-1}\right)=1$.

We also have a factorization

$$
f_{\log }(\mathcal{M}): T_{Q} \times X_{Q} \cong X_{\log }(\mathcal{M}) \stackrel{h_{\log }}{\longrightarrow} X_{\log }(\mathcal{F}) \stackrel{f_{\log }(\mathcal{F})}{\longrightarrow} Y_{\log } \cong T_{P} \times X_{P}
$$

The fiber of $f_{\log }(\mathcal{M})$ over the point $(1,1) \in T_{P} \times X_{P}$ can be identified with $T_{Q / P} \times$ $X_{Q / P}$. Its image in $X_{\log }(\mathcal{F})$ is the fiber of $f_{\log }(\mathcal{F})$ over $(1,1)$, and we denote this image by $A_{Q, P}^{\log }(\mathcal{F})$. Recall the map $\eta_{A, P}^{\prime}: X_{Q} \rightarrow X_{Q}(1) \cong X_{Q / P}$ constructed from an effective generating cycle $A$ for $C_{Q}$ in Corollary 2.12, and consider the following commutative diagram:

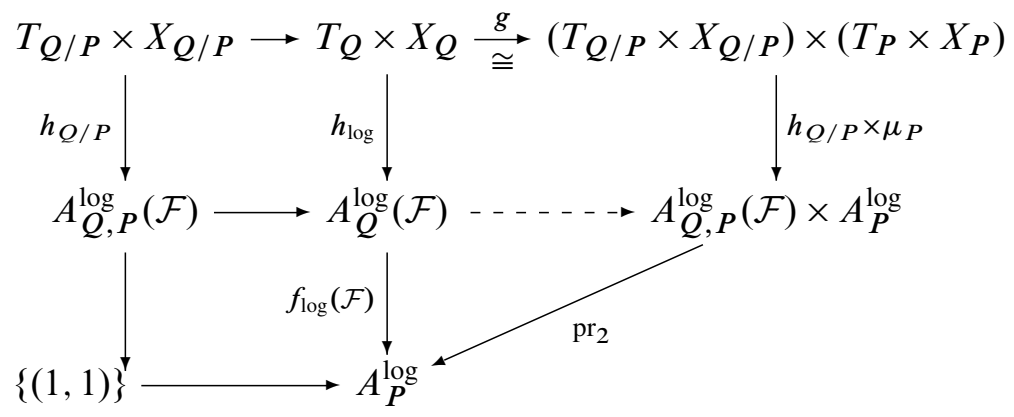

Here the homeomorphism $g$ comes from the splitting $T_{Q} \cong T_{Q / P} \times T_{P}$ induced by $\sigma$ and the homeomorphism $\left(\eta_{A, P}^{\prime}, X_{\theta}\right): X_{Q} \cong X_{Q / P} \times X_{P}$ of Corollary 2.12. The map $\mu_{P}: T_{P} \times X_{P} \rightarrow A_{P}^{\log }$ is the canonical isomorphism, and $h_{Q / P}$ is simply the restriction of $h_{\log }$ to the subset $T_{Q / P} \times X_{Q / P}$ of $T_{Q} \times X_{Q}$. Recall from (2) of Corollary 2.12 that for any $x \in X_{Q}$

$$
\langle F(x)+P\rangle=\left\langle F\left(\eta_{A, P}^{\prime}(x)\right)+P\right\rangle=F\left(\eta_{A, P}^{\prime}(x)\right),
$$

and hence $G(x)=G\left(\eta_{A, P}^{\prime}(x)\right)$. Thus $g$ is compatible with the equivalence relations defined by $h_{\log }$ and $h_{Q / P} \times \mu_{P}$. It follows that there is a homeomorphism filling in the diagram as shown by the dashed arrow. This proves that $f_{\log }(\mathcal{F})$ is a product map, hence a submersion.

It remains only to describe the fiber $A_{Q, P}^{\log }(\mathcal{F})$. Note that $Q / P$ is still a fine monoid, but it need not be sharp-its group of units is identified with $\langle P\rangle / P$. Let $A_{Q / P}(\mathcal{M})$ denote the log space defined by $Q / P$ with the coherent log structure defined by $Q / P$ and let $A_{Q / P}(\mathcal{F})$ be the relatively coherent log space defined by the sheaf of faces generated by $G / P$. Then $A_{Q, P}^{\log }(\mathcal{F})$ can be identified with $A_{Q / P}^{\log }(\mathcal{F})$. It is easy to 
check that the condition (b) is still satisfied by the map $A_{Q / P}(\mathcal{F}) \rightarrow p t$, so this map is again relatively smooth. Thus we are reduced to the case in which $P=0$.

Suppose first that $G=Q^{*}$. In this case $A_{Q}^{\log }(\mathcal{F})=A_{Q}$, and since $\bar{Q}$ is free, the affine toric analytic space $A_{Q}$ is a smooth manifold. If $G \neq Q^{*}$, choose a finite set of generators $\left(g_{1}, \ldots, g_{k}\right)$ for the fine submonoid $G$ of $Q$. Then $G$ is the face of $Q$ generated by $g:=g_{1}+g_{2}+\cdots+g_{k}$. Consider the map $\theta: \mathbf{N} \rightarrow Q$ sending 1 to $g$. This map is injective, local, and locally exact, and the stalks of the quotient $\mathcal{M} / \mathcal{F}$ are free. Thus the corresponding map of log analytic spaces $\tilde{f}:=A_{\theta}:\left(A_{Q}, \mathcal{F}\right) \rightarrow A_{\mathbf{N}}$ is relatively smooth. It follows from what we have proved so far that, after a finite covering of the base, there is an isomorphism $A_{Q}^{\log }(\mathcal{F}) \cong A_{\mathbf{N}}^{\log } \times A_{Q / \mathbf{N}}^{\log }(\mathcal{F})$. But now the image of $G$ in $Q / \mathbf{N}$ is contained in the group of units, so by the previous case, $A_{Q / \mathbf{N}}^{\log }(\mathcal{F})$ is a manifold. Since $A_{\mathbf{N}}^{\log }$ is a manifold with boundary $B:=\tau^{-1}(0)$, it follows that $A_{Q}^{\log }(\mathcal{F})$ is a topological manifold with boundary $\tilde{f}_{\log }^{-1}(B)$. But $\tilde{f}_{\log }^{-1}(B)$ is exactly the subset of $A_{Q}^{\log }(\mathcal{F})$ lying over the locus of $A_{Q}(\mathcal{F})$ where the log structure is not trivial.

\section{Idealized monoids and log spaces}

In this section we describe a generalization of our techniques which may prove useful in analyzing strata of toric varieties and log spaces. The only real subtleties are hidden in the meanings of definitions, so we attempt to explain these carefully, only sketching the parts of the arguments that are parallel to the nonidealized version.

By an idealized monoid we mean a pair $(M, K)$ where $M$ is a (commutative) monoid and $K$ is an ideal of $M$. By a face of $(M, K)$ we mean a face $F$ of $M$ which does not meet $K$, or equivalently, such that the corresponding prime ideal contains $K$. We write $\operatorname{Spec}(M, K)$ for the set of such prime ideals. If $\theta:(P, J) \rightarrow(Q, K)$ is a homomorphism of idealized monoids, let $P^{+} Q$ denote the ideal of $Q$ generated by the image of the maximal ideal $P^{+}$of $P$ and let $K_{\theta}:=\left(P^{+} Q\right) \cup K$. Then $\operatorname{Spec}\left(Q, K_{\theta}\right)$ is the set of prime ideals of $(Q, K)$ lying over $P^{+}$.

Definition 4.1 A homomorphism of idealized monoids $\theta:(P, J) \rightarrow(Q, K)$ is exact if $\theta^{-1}(K)=J$ and for every face $P^{\prime}$ of $(P, J)$, the restriction $\theta^{\prime}$ of $\theta$ to $P^{\prime}$ is exact.

Note that every exact homomorphism is local—it sends the closed point of $\operatorname{Spec}(Q, K)$ (if there is one) to the closed point of $\operatorname{Spec}(P, J)$. Indeed, if $K=Q$ then $\operatorname{Spec}(Q, K)$ is empty and there is nothing to check, so we may assume that $K$ is a proper ideal. Then if $p \in P$ and $\theta(p) \in Q^{*}, \theta(p) \notin K$ so $p \notin J$, and the same is true for every 
multiple of $p$. It follows that $p$ does not belong to the radical of $J$ and hence that there is a face $P^{\prime}$ of $(P, J)$ containing $p$. Since $P^{\prime} \rightarrow Q$ is exact, it is local, and it follows that $p$ is a unit of $P^{\prime}$.

Proposition 4.2 Let $\theta:(P, J) \rightarrow(Q, K)$ be a homomorphism of idealized monoids. Consider the following conditions:

(1) $\theta:(P, J) \rightarrow(Q, K)$ is exact.

(2) $\operatorname{Spec} \theta: \operatorname{Spec}(Q, K) \rightarrow \operatorname{Spec}(P, J)$ is surjective.

Then (1) implies (2), and the converse holds if $P$ and $Q$ are fine and saturated and $J$ and $K$ are radical ideals.

Proof Suppose (1) holds and $F$ is a face of $(P, J)$. It is straightforward to verify that $\theta_{F}:\left(P_{F}, J_{F}\right) \rightarrow\left(Q_{F}, K_{F}\right)$ is still exact and hence is local, as we saw above. Furthermore, since $J=\theta^{-1}(K)$ does not meet $F, K_{F}$ is a proper ideal of $Q_{F}$. Hence $Q_{F}^{*}$ is a face of $\left(Q_{F}, K_{F}\right)$ and its inverse image $G$ in $Q$ is a face of $(Q, K)$ above $F$. This proves the surjectivity of $\operatorname{Spec} \theta$. Conversely, suppose that (2) holds and that $P$ and $Q$ are fine and saturated. Let $F$ be a face of $(P, J)$, and choose a face $G$ of $(Q, K)$ lying over $F$. Every face $F^{\prime}$ of $F$ is a face of $(P, J)$, so there is a face $G^{\prime}$ of $(Q, K)$ lying over $F^{\prime}$. Then $G \cap G^{\prime}$ is a face of $G$ lying over $F^{\prime}$. This shows that Spec $G \rightarrow \operatorname{Spec} F$ is surjective. Since $F$ and $G$ are fine and saturated, it follows that $F \rightarrow G$ is exact. Since $G$ is a face of $Q, G \rightarrow Q$ is exact, and it follows that $F \rightarrow Q$ is also exact. Now since $J$ is a radical ideal, it is the intersection of all the primes $\mathfrak{p}$ of $\operatorname{Spec}(P, J)$, and since each such prime comes from a prime of $Q$ containing $K$, $J=\theta^{-1}(K)$.

Definition 4.3 A morphism of idealized monoids $\theta:(P, J) \rightarrow(Q, K)$ is

(1) locally exact if for every face $G$ of $(Q, K)$, the map $\left(P_{F}, J_{F}\right) \rightarrow\left(Q_{G}, K_{G}\right)$ is exact, where $F=\theta^{-1}(G)$,

(2) very locally exact if for every face $G$ of $\left(Q, K_{\theta}\right)$, the map $(P, J) \rightarrow\left(Q_{G}, K_{G}\right)$ is exact.

Lemma 4.4 Let $\theta:(P, J) \rightarrow(Q, K)$ be a local and very locally exact homomorphism of fine saturated idealized monoids.

(1) For every face $G$ of $\operatorname{Spec}(Q, K)$, the induced map

$$
F:=\theta^{-1}(G) \rightarrow G
$$

is locally exact.

(2) $\theta$ is locally exact. 
Proof Let $G$ be a face of $(Q, K)$ and $F:=\theta^{-1}(G)$. Let $G^{\prime}$ be a face of $G$ with $\theta^{-1}\left(G^{\prime}\right)=P^{*}$. Then $G^{\prime}$ is a face of $\left(Q, K_{\theta}\right)$ and by assumption $(P, J) \rightarrow\left(Q_{G^{\prime}}, K_{G^{\prime}}\right)$ is exact. Since $F$ is a face of $(P, J)$, the composite $F \rightarrow Q_{G^{\prime}}$ is exact, and hence $F \rightarrow G_{G^{\prime}}$ is also exact. Since this is true for every face $G^{\prime}$ of $G$ with $\theta^{-1}\left(G^{\prime}\right)=P^{*}$, $F \rightarrow G$ is very locally exact. By Theorem 2.3 it is locally exact. This proves (1).

It will suffice to replace $J$ and $K$ by their radicals and to work with the associated cones. Again let $G$ be a face of $(Q, K)$ and $F:=\theta^{-1}(G)$; we claim that $\left(P_{F}, J_{F}\right) \rightarrow$ $\left(Q_{G}, K_{G}\right)$ is exact. First we show that if $P^{\prime}$ is any face of $(P, J)$ containing $F$, then $P_{F}^{\prime} \rightarrow Q_{G}$ is exact. Suppose that $a \in P^{\prime \text { gp }}$ and $\theta(a) \in Q_{G}$. Then there exists some $g \in G$ with $g+\theta(a) \in Q$. Since we are working with cones, we can apply Theorem 2.3 to the locally exact map $\theta_{F}: F \rightarrow G$ induced by $\theta$. Thus we can write $g=\theta(f)+g^{\prime}$, where $g^{\prime}$ is contained in a face $G^{\prime}$ of $\left(G, P^{+} G\right)$ and $f \in F$. Since $G$ is a face of $(Q, K)$, in fact $G^{\prime}$ is a face of $\left(Q, K_{\theta}\right)$, and since $\theta$ is very locally exact, $(P, J) \rightarrow\left(Q_{G^{\prime}}, K_{G^{\prime}}\right)$ is exact. In particular, $P^{\prime} \rightarrow Q_{G^{\prime}}$ is exact. Since $\theta(a+f) \in Q_{G^{\prime}}$, $a+f \in P^{\prime}$ and hence $a \in P_{F}^{\prime}$, as required. Finally, we claim that the inverse image of $K_{G}$ in $P_{F}$ is $J_{F}$. Indeed, if $\theta(p-f)=k-g$, then we can use the argument above to write $g=g^{\prime}+\theta\left(f^{\prime}\right)$, where $\theta^{-1}\left\langle g^{\prime}\right\rangle=F^{*}$. Since $(P, J) \rightarrow\left(Q_{G^{\prime}}, K_{G^{\prime}}\right)$ is exact, it follows that $p-f \in J_{F}$.

Note that the homomorphism

$$
\theta:(\mathbf{N}, \varnothing) \rightarrow\left(\mathbf{N} \oplus \mathbf{N}, \mathbf{N}^{+} \oplus \mathbf{N}^{+}\right), \quad n \mapsto(n, 0)
$$

satisfies condition (1) but it is not very locally exact.

If $(Q, K)$ is an idealized monoid, let

$$
C_{Q}(K):=\bigcup\left\{C_{G}: G \text { is a face of }(Q, K)\right\},
$$

and if $\theta:(P, J) \rightarrow(Q, K)$ is a homomorphism of idealized monoids, let

$$
C_{Q, P}(K):=\bigcup\left\{C_{G}: G \text { is a face of }\left(Q, K_{\theta}\right)\right\} \text {. }
$$

Proposition 4.5 Let $\theta:(P, J) \rightarrow(Q, K)$ be a local and locally exact homomorphism of fine idealized monoids. Assume that $P$ is sharp. Then the addition map induces a homeomorphism

$$
\sigma: C_{Q, P}(K) \times C_{P}(J) \rightarrow C_{Q}(K) .
$$

For each pair $(F, G)$, where $F$ is a face of $(P, J)$ and $G$ a face of $\left(Q, K_{\theta}\right),\langle F+G\rangle$ is a face of $(Q, K)$, and $\sigma$ induces a homeomorphism

$$
C_{\langle F+G\rangle, F} \times C_{G} \rightarrow C_{\langle F+G\rangle} .
$$


Proof We first check that the addition map sends $C_{Q, P}(K) \times C_{P}(J)$ to $C_{Q}(K)$. If $q \in C_{Q, P}(K)$ there exists a face $G$ of $\left(Q, K_{\theta}\right)$ containing $q$, and if $p \in C_{P}(J)$ there exists a face $F$ of $(P, J)$ containing $p$. Since $\theta_{G}:(P, J) \rightarrow\left(Q_{G}, K_{G}\right)$ is exact, $\operatorname{Spec}\left(\theta_{G}\right)$ is surjective, so there exists a face $G^{\prime}$ of $(Q, K)$ containing $G$ such that $\theta^{-1}\left(G^{\prime}\right)=F$. Then $G^{\prime}$ contains $\theta(p)+q$, and hence $\theta(p)+q \in C_{Q}(K)$, as required. To see that $\sigma$ is injective, suppose that $\left(q_{i}, p_{i}\right) \in C_{Q, P}(K) \times C_{P}(J)$ for $i=1,2$, and $q_{1}+\theta\left(p_{1}\right)=q_{2}+\theta\left(p_{2}\right)$. Let $G^{\prime}$ be the face of $C_{Q}$ generated by $\theta\left(p_{i}\right)+q_{i}$; note that $\theta\left(p_{i}\right)$ and $q_{i}$ belong to $G^{\prime}$. We have seen that $G^{\prime}$ is a face of $(Q, K)$, so $F^{\prime}:=\theta^{-1}\left(G^{\prime}\right)$ is a face of $(P, J)$ and $F^{\prime} \rightarrow G^{\prime}$ is locally exact, by Lemma 4.4. Then it follows from Theorem 2.3 that $q_{1}=q_{2}$ and $p_{1}=p_{2}$. For the surjectivity, suppose that $q \in C_{Q}(K)$. Then there is a face $G$ of $(Q, K)$ containing $q$, and $F:=\theta^{-1}(G)$ is a face of $(P, J)$. By Lemma 4.4, $F \rightarrow G$ is locally exact, and it follows from Theorem 2.3 that there exist an element $q^{\prime}$ of $G$ with $\theta^{-1}\left\langle q^{\prime}\right\rangle=\{0\}$ and an element $p$ of $F$ such that $q^{\prime}+\theta(p)=q$. Then $\left(q^{\prime}, p\right) \in C_{Q, P}(K) \times C_{P}(J)$ and $\sigma\left(q^{\prime}, p\right)=q$ as required. Finally, since $C_{Q}(K)$ admits a locally closed finite cover by the sets $C_{G}$ as $G$ ranges over the faces of $(Q, K)$ and the restriction of $\sigma^{-1}$ to each of these is continuous, it follows that $\sigma^{-1}$ is also continuous. The compatibility expressed in the last statement is clear.

Now we can prove the analog of the main local rounding result (Theorem 2.5). If $(M, K)$ is an idealized monoid, we write $X_{M}(K)$ for the set of elements $x$ of $X_{M}$ which annihilate $K$. Suppose that $\theta:(P, J) \rightarrow(Q, K)$ is as in Proposition 4.5, and let

$$
\pi:=\operatorname{pr}_{1} \circ \sigma^{-1}: C_{Q}(K) \rightarrow C_{Q, P}(K) \times C_{P}(J) \rightarrow C_{Q, P}(K) .
$$

Choose an effective generating cycle $A$ for $C_{Q}$. Then the associated moment map $\mu: X_{Q} \rightarrow C_{Q}$ induces maps $X_{Q}(K) \rightarrow C_{Q}(K)$ and $X_{Q, P}(K) \rightarrow C_{Q, P}(K)$, where $X_{Q, P}(K):=X_{Q}\left(K_{\theta}\right)$. Let $v: C_{Q, P}(K) \rightarrow X_{Q, P}(K)$ denote the inverse of the latter, and let $\eta$ be the composite

$$
\eta: X_{Q}(K) \stackrel{\mu_{A}}{\longrightarrow} C_{Q}(K) \stackrel{\pi}{\longrightarrow} C_{Q, P}(K) \stackrel{\nu_{A, P}}{\longrightarrow} X_{Q, P}(K) .
$$

Proposition 4.6 With the notation and hypotheses above, the map

$$
\left(\eta, X_{\theta}\right): X_{Q}(K) \rightarrow X_{Q, P}(K) \times X_{P}(J)
$$

is a homeomorphism.

Proof If $Q^{\prime}$ is a face of $(Q, K)$, let $P^{\prime}:=\theta^{-1}\left(Q^{\prime}\right)$ and let $\theta^{\prime}: P^{\prime} \rightarrow Q^{\prime}$ be the homomorphism induced by $\theta$. Then $\theta^{\prime}$ is locally exact and local, and the restriction 
of $A$ to $Q^{\prime}$ is an effective generating cycle for $Q^{\prime}$. The map above then restricts to a map

$$
\left(\eta^{\prime}, X_{\theta^{\prime}}\right): X_{Q^{\prime}} \rightarrow X_{Q^{\prime}, P^{\prime}} \times X_{P^{\prime}},
$$

which is a homeomorphism by Theorem 2.5. To see that $\left(\eta, X_{\theta}\right)$ is injective, let $x_{1}$ and $x_{2}$ be two points of $X_{Q}(K)$ with the same image $(y, z)$ in $X_{Q, P}(K) \times X_{P}(J)$. Choose faces $Q_{i}$ of $(Q, K)$ with $x_{i} \in X_{Q_{i}}$ and let $P_{i}:=\theta^{-1}\left(Q_{i}\right)$. Since $X_{Q_{i}}$ is invariant under $\eta, y \in X_{Q_{i}}$. It follows that $y$ belongs to $X_{Q_{1}} \cap X_{Q_{2}}=X_{Q^{\prime}}$, where $Q^{\prime}:=Q_{1} \cap Q_{2}$. Similarly, $z \in X_{P^{\prime}}$, where $P^{\prime}:=P_{1} \cap P_{2}$. Since the map

$$
\left(\eta^{\prime}, X_{\theta^{\prime}}\right): X_{Q^{\prime}} \rightarrow X_{Q^{\prime}, P^{\prime}} \times X_{P^{\prime}}
$$

is surjective, there is a point $x^{\prime} \in X_{Q^{\prime}}$ mapping to $(y, z)$. Since the restriction of $\left(\eta, X_{\theta}\right)$ to each $X_{Q_{i}}$ is injective, it follows that $x_{1}=x^{\prime}=x_{2}$. This proves the injectivity. To prove surjectivity, let $y$ be a point of $X_{Q, P}(K)$ and $z$ a point of $X_{P}(J)$, and choose a face $G$ of $\left(Q, K_{\theta}\right)$ with $y \in X_{G}$ and a face $F$ of $(P, J)$ with $z \in X_{F}$. As we have seen, there is a face $Q^{\prime}$ of $(Q, K)$ containing $F+G$. Let $P^{\prime}:=\theta^{-1}\left(Q^{\prime}\right)$ and let $\theta^{\prime}: P^{\prime} \rightarrow Q^{\prime}$ be the map induced by $\theta$. Since $(y, z) \in X_{Q^{\prime}, P^{\prime}} \times X_{P^{\prime}} \subseteq X_{Q, P}(K) \times X_{P}(J)$, Theorem 2.5 implies that there is a point of $X_{Q^{\prime}} \subseteq X_{Q}(K)$ mapping to $(y, z)$. The fact that the now bijective map $\left(\eta, X_{\theta}\right)$ is a homeomorphism follows from the fact that its restriction to a finite closed cover is.

If $(Q, K)$ is an idealized monoid, we let $A_{Q}(K)$ denote the closed subspace of $A_{Q}$ defined by the ideal of $\mathbf{C}[Q]$ generated by $K$. Then we have a proper surjective map

$$
A_{Q}^{\log }(K):=X_{Q}(K) \times T_{Q} \rightarrow A_{Q}(K),
$$

and we immediately obtain the following analog of Proposition 3.2.

Proposition 4.7 Let $\theta:(P, J) \rightarrow(Q, K)$ be a locally exact and injective homomorphism of fine idealized monoids. Then the corresponding map

$$
A_{\theta}^{\log :} A_{Q}^{\log }(K) \rightarrow A_{P}^{\log }(J)
$$

is a topological submersion.

An idealized log analytic space is a log analytic space $X$ endowed with a sheaf of ideals $\mathcal{K}_{X}$ in the sheaf of monoids $\mathcal{M}_{X}$ such that $\alpha_{X}(k)=0$ for every local section $k$ of $\mathcal{K}_{X}$. A morphism $f: X \rightarrow Y$ of idealized log spaces is required to be compatible with the ideals, so that $f^{\text {b }}$ maps $f^{-1} \mathcal{K}_{Y}$ to $\mathcal{K}_{X}$. The category of idealized log analytic spaces such that $\mathcal{K}_{X}$ is empty is equivalent to the usual category of log analytic spaces. An idealized $\log$ analytic space is fine if $\mathcal{M}_{X}$ is fine and the sheaf of ideals $\mathcal{K}_{X}$ is locally generated by a finite set of sections. 
Definition 4.8 A morphism $f: X \rightarrow Y$ of fine idealized log analytic spaces is exact if for every $x \in X$, the map

$$
f_{x}^{b}:\left(\mathcal{M}_{Y, f(x)}, \mathcal{K}_{Y, f(x)}\right) \rightarrow\left(\mathcal{M}_{X, x}, \mathcal{K}_{X, x}\right)
$$

is exact.

It follows from Proposition 4.2 that if $\mathcal{M}_{X}$ and $\mathcal{M}_{Y}$ are fine and saturated and $\mathcal{K}_{X}$ and $\mathcal{K}_{Y}$ are radical ideals, then $f$ is exact if and only if for every $x \in X$, the map

$$
\operatorname{Spec}\left(f_{x}^{b}\right): \operatorname{Spec}\left(\mathcal{M}_{X, x}, \mathcal{K}_{X, x}\right) \rightarrow \operatorname{Spec}\left(\mathcal{M}_{Y, f(x)}, \mathcal{K}_{Y, f(x)}\right)
$$

is surjective.

The following result generalizes Theorem 3.5. We do not give a detailed general treatment of smoothness for morphisms of idealized log spaces here, just taking as a definition the existence of local charts as in the course of the proof below.

Theorem 4.9 Let $f: X \rightarrow Y$ be a smooth and exact morphism of fine idealized log analytic spaces. Then $f_{\log }: X_{\log } \rightarrow Y_{\log }$ is a topological submersion.

Proof We may and shall assume without loss of generality that $\mathcal{K}_{X}$ and $\mathcal{K}_{Y}$ are radical ideals. The statement is local on $X$ and $Y$, and the smoothness of $f$ then implies that, locally on $X$ and $Y$, there exist a homomorphism of idealized monoids $\theta:(P, J) \rightarrow(Q, K)$ and a diagram:

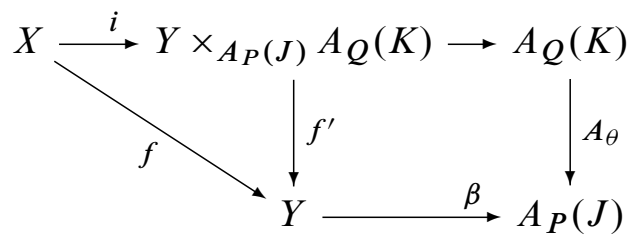

Here again the square is Cartesian and $i$ is a strict open immersion, and furthermore the ideals $\mathcal{K}_{X}$ and $\mathcal{K}_{Y}$ are generated by $K$, and $J$, respectively. If $f$ is exact, one can conclude as in the proof of Theorem 3.5 that $\theta$ is very locally exact and hence locally exact. Then the Theorem follows from Proposition 4.6.

\section{Complements and applications}

In this section we give some applications of our results to log geometry. Many of these have already been envisioned, and some also proved. In particular, Usui proved Theorem 5.1 and its Corollary 5.2 when $f$ is a multigeneralized semistable family over a polydisk [19]. Later, in [9], Kajiwara and Nakayama suggested Theorems 3.5 
and 5.1 and managed to prove Corollary 5.2 in the coherent case without the use of either of these results.

Theorem 5.1 Let $f: X \rightarrow Y$ be a morphism of $\log$ analytic spaces, where $Y$ is fine and $X$ is relatively coherent. Assume that $f$ is proper, separated, exact, and relatively smooth. Then the map $f_{\log }: X_{\log } \rightarrow Y_{\log }$ is a topological fiber bundle whose fibers are oriented manifolds with boundary. That is, locally on $Y_{\log }$, it is homeomorphic to a projection mapping $Z \times Y_{\log } \rightarrow Y_{\log }$, where $Z$ is an oriented manifold with boundary.

Proof Theorem 3.7 tells us that $f_{\log }$ is a submersion whose fibers are manifolds with boundary, and Siebenmann's [18, Corollary 6.14] asserts that a proper separated topological submersion whose fiber is stratifiable is in fact a fiber bundle. Since a manifold with boundary has a 2 -step stratification, we can conclude that $f_{\log }$ is a fiber bundle. We will discuss the orientation in Theorem 5.10.

Corollary 5.2 With the hypotheses of Theorem 5.1, let $F$ be a locally constant abelian sheaf on $X_{\log }$. Then for all integers $q, R^{q} f_{\log *}(F)$ is locally constant on $Y_{\log }$.

Proof This follows easily from Theorem 5.1, as explained in [9, Remark B.2.1]. However it may be of some interest to provide a proof which does not depend on Siebenmann's theorem and also does not use a stratification of the fiber. For this we can appeal to the elementary argument outlined in the appendix and Proposition 5.3 below. This argument also shows that the corollary is true even if condition (2b) of Definition 3.6 is not satisfied. (See the remark after Definition 3.6.)

Proposition 5.3 Let $Y$ be a fine $\log$ analytic space. Then $Y_{\log }$ is locally triangulizable. In particular, it is locally path connected and semilocally simply connected.

Proof First suppose that $Y=A_{P}$ with its standard log structure. Then $Y_{\log }=X_{P} \times T_{P}$. Here $X_{P}$ and $T_{P}$ are semialgebraic subsets of $\mathbf{R}^{n}$, and the map $X_{P} \times T_{P} \rightarrow A_{P}$ is algebraic. Since semialgebraic sets are triangulizable, as Hironaka showed in [6], the result is certainly true in this case.

Our general statement is local, so we may assume that there is a fine chart $P \rightarrow M_{Y}$ for $Y$. Such a chart defines a strict morphism of log analytic varieties $Y \rightarrow A_{P}$, and hence a Cartesian diagram:

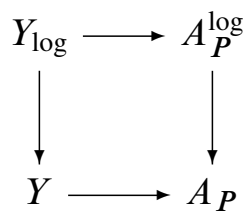

Since the maps $Y \rightarrow A_{P}$ and $A_{P}^{\log } \rightarrow A_{P}$ are analytic maps, the fiber product is semianalytic subset of a suitable affine space, and hence also locally triangulizable by [6]. 
The fiber bundles in Theorem 5.1 can be used to give geometric models of nearby cycles and monodromy. The following result is a first step in this direction; we expect more explicit results could be constructed in many situations.

To motivate the discussion, consider the case of a smooth proper morphism of analytic spaces $f^{*}: X^{*} \rightarrow D^{*}$, where $D^{*}$ is the punctured disk. Then $f^{*}$ is a fiber bundle, typically nontrivial, and one wants to relate the action of the monodromy group $\pi_{1}\left(D^{*}\right)$ on the generic fiber to the degeneration of $f^{*}$. Suppose that $f^{*}$ can be extended to a relatively smooth proper map of $\log$ analytic spaces $f: X \rightarrow D$, where $D$ is the standard $\log$ disk. Then the inclusion $D^{*} \rightarrow D_{\log }$ is a homotopy equivalence, and the logarithmic model $f_{\log }: X_{\log } \rightarrow D_{\log }$ compactifies $f^{*}$ and remains a fiber bundle. In fact if $P \rightarrow D$ is the origin (so that $P$ is a log point), then $P_{\log } \cong \mathbf{S}^{1}$, the inclusion $P_{\log } \rightarrow D_{\log }$ is also a homotopy equivalence, and the restriction of $f$ to the special fiber $X_{0} \rightarrow P$ determines the bundle $X_{0}^{\log } \rightarrow P_{\log }$ and hence the monodromy of $X_{\log } \rightarrow D_{\log }$ and of $X^{*} \rightarrow D^{*}$. Thus it is sensible to restrict attention to smooth maps over log points.

In fact we shall work over a general fine saturated $\log$ point $P$. Then $P_{\log }$ is canonically isomorphic to $\operatorname{Hom}\left(\bar{M}_{P}, \mathbf{S}^{1}\right)$, and its universal cover $\widetilde{P}_{\log } \rightarrow P_{\log }$ is given by the exponential map

$$
\operatorname{Hom}\left(\bar{M}_{P}, \mathbf{R}(1)\right) \rightarrow \operatorname{Hom}\left(\bar{M}_{P}, \mathbf{S}^{1}\right) .
$$

Thus the log inertia group of $P$, ie, the fundamental group $\pi_{1}\left(P_{\log }\right)$, is $\operatorname{Aut}\left(\widetilde{P}_{\log } / P_{\log }\right)$ which is canonically identified with $\operatorname{Hom}\left(\bar{M}_{P}, \mathbf{Z}(1)\right)$. Now let $f: Y \rightarrow P$ be a relatively smooth saturated and exact morphism of log analytic spaces. (For the definition and a discussion of saturated morphisms, we refer to the appendix of [7].) Then $f_{\log }: Y_{\log } \rightarrow P_{\log }$ is a submersion. Let us consider the Cartesian diagram:

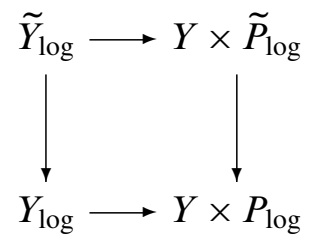

The map $\psi: \tilde{Y}_{\log } \rightarrow Y \times \widetilde{P}_{\log } \rightarrow Y$ is called the nearby cycle map. Since $f$ is saturated, the set $Y^{\text {st }}$ where $f$ is strict is dense and open in $Y$, and the map

$$
\psi_{\mathrm{st}}: Y_{\log }^{\mathrm{st}} \rightarrow Y^{\mathrm{st}} \times P_{\log }
$$

is an isomorphism. Thus the submersion $f_{\log }$ is canonically trivialized over the open set $Y_{\log }^{\text {st }}$. 
Proposition 5.4 Let $f: Y \rightarrow P$ be a relatively smooth, saturated, exact, proper, and separated morphism of $\log$ analytic spaces, where $P$ is a $\log$ point. Let $Z$ be the fiber of $f_{\log }$ over the origin of $P_{\log }$, and let $Y^{\prime}$ be any compact subset of $Y^{\text {st }}$. Then there exists a trivialization $\tilde{Y}_{\log } \cong Z \times \widetilde{P}_{\log }$ whose restriction to $\tilde{Y}_{\log }^{\prime}:=Y^{\prime} \times \widetilde{P}_{\log }$ agrees with the canonical trivialization described above.

Proof Identify $\bar{M}_{P}^{\text {gp }}$ with $\mathbf{Z}_{>}$and $\widetilde{P}_{\log }$ with $\mathbf{R}(1)^{r}$. Choose $n \in \mathbf{N}^{+}$, let $B:=$ $[-2 n \pi i, 2 n \pi i]^{r}$, and restrict everything to $B$. Then the fiber bundle $\tilde{Y}_{\log } \rightarrow B$ can be trivialized, and we may choose a trivialization $\Phi: \tilde{Y}_{\log } \cong Z \times B$. Then $Y^{\text {st }}$ becomes an open subset of $Z$, and the composite

$$
\phi: Y^{\mathrm{st}} \times B \stackrel{\psi_{\mathrm{st}}^{-1}}{\longrightarrow} \tilde{Y}_{\mathrm{log}} \stackrel{\Phi}{\longrightarrow} Z \times B, \quad(y, t) \mapsto\left(\phi_{t}(y), t\right)
$$

defines a family of open immersions $\left\{\phi_{t}: t \in B\right\}$, where $\phi_{0}$ is just the inclusion $Y^{\text {st }} \subseteq Z$. By Siebenmann's isotopy extension theorem [18, 6.5], there is a homeomorphism $\Phi^{\prime}: Z \times B \rightarrow Z \times B$ (over $B$ ) whose restriction to $\tilde{Y}_{\log }^{\prime} \times B$ is $\left.\phi\right|_{Y^{\prime}}$. Applying [18, 6.5 , III] we can extend $\Phi^{\prime}$ to sets $B$ with larger and larger $n$, obtaining the statement of the proposition.

For each $\theta \in B$, the proposition determines an isomorphism $Z \cong f_{\log }^{-1}\left(e^{\theta}\right)$. In particular if $\gamma \in \pi_{1}\left(P_{\log }\right)$, one obtains isomorphisms $Z \cong Y_{1}^{\log }:=f_{\log }^{-1}(1)$, which differ by an automorphism $T_{\gamma}$ of $Y_{1}^{\log }$. Note that the restriction of $T_{\gamma}$ to the complement of a suitable neighborhood of the nonstrict locus is the identity. Thus the monodromy "lives near the log structure."

Our next goal is to discuss orientation and duality. First we need some preliminary remarks. If $X$ is a fine $\log$ analytic space, $X_{\log }$ is in general just a topological space. However, over the open set $X^{*}$ of $X$ where the log structure is trivial, the map $X_{\log }^{*} \rightarrow X^{*}$ is an isomorphism, so $X_{\log }^{*}$ inherits a complex analytic structure. Furthermore, if $X / \mathbf{C}$ is smooth (in the $\log$ sense), $X^{*}$ is dense in $X$. If $f: X \rightarrow Y$ and $y$ is a point of $Y_{\log }$, then the fiber $X_{y}^{\log }$ of $f_{\log }$ over $y$ is again just a topological space. However, the map $\tau: X_{y}^{\log } \rightarrow X_{\tau(y)}$ is an isomorphism over the strict locus of $f$. Thus $\tau_{X}^{-1} X_{\tau(y)}^{\text {st }}$ has a complex analytic structure, but this set need not be dense, even if $f$ is smooth and exact. We will show that in this case there is a larger open subset of $X_{\log }$ whose intersection with every fiber is dense and endowed with a natural complex analytic structure and hence a natural orientation.

Definition 5.5 A morphism $\theta: P \rightarrow Q$ of integral monoids is small if

$$
\operatorname{Cok}\left(\theta^{\mathrm{gp}}\right): P^{\mathrm{gp}} \rightarrow Q^{\mathrm{gp}}
$$

is a torsion group. A morphism of $\log$ spaces $f: X \rightarrow Y$ is small if for every $x \in X$, the morphism $\bar{M}_{Y, f(x)}^{\mathrm{gp}} \rightarrow \bar{M}_{X, x}^{\mathrm{gp}}$ is small. 
Lemma 5.6 Let $\theta: P \rightarrow Q$ be a morphism of fine monoids.

(1) If $\bar{\theta}: \bar{P} \rightarrow \bar{Q}$ is small, then $\theta$ is vertical.

(2) If $\bar{\theta}$ is exact and small, then for every $q \in Q$, there exist a positive integer $n$, a $u \in Q^{*}$, and a $p \in P$ such that $n q=u+\theta(p)$.

Proof There is an exact sequence

$$
\operatorname{Cok}\left(\theta^{*}\right) \rightarrow \operatorname{Cok}\left(\theta^{\mathrm{gp}}\right) \rightarrow \operatorname{Cok}\left(\bar{\theta}^{\mathrm{gp}}\right) \rightarrow 0 .
$$

If $\bar{\theta}$ is small, $\operatorname{Cok}\left(\bar{\theta}^{\mathrm{gp}}\right)$ is a finite group, and hence the image $\bar{Q} / \bar{P}$ of $\bar{Q}$ in $\operatorname{Cok}\left(\bar{\theta}^{\mathrm{gp}}\right)$ is a finite integral monoid, hence a group. It follows that $Q / P$ is also a group, so that $\theta$ is vertical. If $\bar{\theta}$ is exact, so is $\theta$. For any $q \in Q$, there exist a positive integer $n$, a unit $u$ of $Q$, and elements $p_{1}, p_{2}$ of $P$ such that $n q+u=\theta\left(p_{2}\right)-\theta\left(p_{1}\right)$. Since $\theta$ is exact, it follows that there is some $p \in P$ such that $\theta\left(p_{2}\right)-\theta\left(p_{1}\right)=\theta(p)$.

Proposition 5.7 Let $P \rightarrow Q$ be a local and locally exact homomorphism of fine monoids, with $P$ sharp. Then for every face $G$ of $Q, G \cap P=\{0\}$ if and only if $G^{\mathrm{gp}} \cap P^{\mathrm{gp}}=\{0\}$. Moreover, if $G$ is such a face, the following are equivalent.

(1) $G$ is maximal among all faces of $Q$ such that $G \cap P=\{0\}$.

(2) $P \rightarrow Q / G$ is small.

(3) The natural map $C_{G}^{\mathrm{gp}} \oplus C_{P}^{\mathrm{gp}} \rightarrow C_{Q}^{\mathrm{gp}}$ is an isomorphism.

We shall call a face $G$ satisfying the above conditions cosmall.

Proof Let us first note that when $P \rightarrow Q$ is injective and locally exact and $G$ is a face of $Q, G \cap P=\{0\}$ if and only if $G^{\mathrm{gp}} \cap P^{\mathrm{gp}}=\{0\}$. Indeed, if $G \cap P=\{0\}$ and $g_{i} \in G, p_{i} \in P$, with $g_{1}-g_{2}=p_{1}-p_{2}$, then since $P \rightarrow Q_{G}$ is exact, $p_{1}-p_{2}$ belongs to $P$ and hence also to $G$, hence is zero. Next note that since $P \rightarrow Q$ is locally exact, so is the map $P \rightarrow Q / G$, and since $G^{\mathrm{gp}} \cap P^{\mathrm{gp}}=\{0\}$, the map $P \rightarrow Q^{\prime}:=Q / G$ is still injective. Corollary 2.4 applies to $C_{P} \rightarrow C_{Q^{\prime}}$, and we can conclude that the summation map $C_{Q^{\prime}, P} \times C_{P} \rightarrow C_{Q^{\prime}}$ is bijective. Now suppose that (1) holds, let $q$ be an element of $Q$, and let $q^{\prime}$ be its image in $Q^{\prime}$. Then there exist a face $G^{\prime}$ of $Q^{\prime}$ such that $G^{\prime \mathrm{gp}} \cap P^{\mathrm{gp}}=\{0\}$ and elements $g^{\prime} \in C_{G^{\prime}}$ and $p \in C_{P}$ such that $q^{\prime}=p+g^{\prime}$. The inverse image of $G^{\prime}$ in $Q$ is a face $G^{\prime \prime}$ of $Q$ such that $G^{\prime \prime g p} \cap P^{\mathrm{gp}}=\{0\}$, and hence $G^{\prime \prime}=G$. Thus $g^{\prime}=0$. This shows that $C_{P} \rightarrow C_{Q / G}$ is surjective, and hence that $P \rightarrow Q / G$ is small. Thus (1) implies condition (2), which is clearly equivalent to (3). Furthermore, if $P \rightarrow Q / G$ is small and injective, then the faces of $Q$ containing $G$ correspond bijectively to the faces of $P$, and in particular $G$ is maximal among those faces of $Q$ which meet $P$ in $\{0\}$. 
Proposition 5.8 Let $f: X \rightarrow Y$ be an exact and smooth morphism of fine log analytic spaces.

(1) The set $X^{\text {sm }}$ of points $x$ in $X$ where $f$ is small is open and dense in every fiber.

(2) Suppose $f$ is small. Then for each point $y$ of $Y$, the reduced fiber $X_{y_{\text {red }}}$ is smooth, and the natural map $X_{y}^{\log } \rightarrow X_{y_{\text {red }}}$ is a finite covering space. In particular, there is a unique complex analytic structure on $X_{y}^{\log }$ such that this map is complex analytic, and $X_{y}^{\log }$ has a natural orientation.

Proof Statement (1) is local on $X$, so by the argument at the beginning of the proof of Theorem 3.5, we may assume that $f$ is given by a local and locally exact morphism of fine monoids $\theta: P \rightarrow Q$, where $P$ is sharp. We view $\theta$ as an inclusion. The fiber of Spec $Q \rightarrow$ Spec $P$ over $P^{+}$is the closed subset defined by the ideal $J$ of $Q$ generated by $P^{+}$, and consists of those primes $\mathfrak{p}$ of $Q$ such that $\mathfrak{p} \cap P=P^{+}$. These are the primes corresponding to the faces $G$ of $Q$ such that $G \cap P=\{0\}$, or, by Proposition 5.7, such that $G^{\mathrm{gp}} \cap P^{\mathrm{gp}}=\{0\}$. By Proposition 5.7, the cosmall faces of $Q$ correspond exactly to the minimal primes of $Z(J)$. Thus the set of all such primes is dense and open in $Z(J)$. Statement (1) follows.

Replace $Q$ by $Q_{G}$, so that $P \rightarrow \bar{Q}$ is small. Then if $q \in Q^{+}$, there is an $n \in \mathbf{Z}_{>}$such that $n q \in J$. It follows that the radical of $J$ is $Q^{+}$, and hence that the reduced fiber of $A_{Q} \rightarrow A_{P}$ is $A_{Q^{*}}$, which is smooth. Furthermore, the splitting $C_{Q} \cong C_{\bar{Q}} \times C_{Q^{*}}$ makes it clear that $X_{Q, P} \cong X_{Q^{*}}$. Moreover, $Q^{*} \cap P^{\mathrm{gp}}=\{0\}$, so we have exact sequences

$$
\begin{gathered}
0 \rightarrow Q^{*} \rightarrow Q^{\mathrm{gp}} / P^{\mathrm{gp}} \rightarrow \bar{Q}^{\mathrm{gp}} / P^{\mathrm{gp}} \rightarrow 0 \\
0 \rightarrow T_{\bar{Q} / P} \rightarrow T_{Q / P} \rightarrow T_{Q^{*}} \rightarrow 0,
\end{gathered}
$$

and the map $T_{Q / P} \rightarrow T_{Q^{*}}$ is a covering space. Thus the fiber of $A_{Q}^{\log } \rightarrow A_{P}^{\log }$ is $X_{Q^{*}} \times T_{Q / P}$, which maps to $X_{y_{\text {red }}}=A_{Q^{*}} \cong X_{Q^{*}} \times T_{Q^{*}}$ in the evident way. This proves (2), at least locally on $X$. But we already know that $\tau: X_{\log } \rightarrow X$ is proper, and it follows that $X_{y}^{\log } \rightarrow X_{y_{\text {red }}}$ is a covering space.

Remark 5.9 Suppose in the situation of Proposition 5.8 that the log structure $\mathcal{F} \rightarrow \mathcal{O}_{X}$ is only relatively coherent and that $f$ is exact and relatively smooth. Then we can find an open subset $U$ of $X$ which is dense in every fiber and such that $\mathcal{F}_{\left.\right|_{U}}$ is coherent and $U \rightarrow Y$ is small. To prove this we may replace $X$ by an open subset on which $\mathcal{F} \rightarrow \mathcal{O}_{X}$ is relatively coherent in a $\log$ structure $\mathcal{M} \rightarrow \mathcal{O}_{X}$. Furthermore we may assume that $X(\mathcal{M}) \rightarrow Y$ admits a chart subordinate to an exact and locally exact morphism $P \rightarrow Q$ as in the proof of Theorem 3.7. Then the small locus of $X(\mathcal{M}) \rightarrow Y$ satisfies the desired conditions. Indeed, this locus is open and dense in every fiber. Furthermore, if 
$X(\mathcal{M}) \rightarrow Y$ is small at a point $x$ of $X$, then it is vertical, so $\mathcal{F}_{x}=\mathcal{M}_{x}$. The set $U$ may not be unique, so we call it a coherent small locus of $f$.

We are now ready for a discussion of orientation and Poincaré-Verdier duality. We shall use the formulation in the textbook [10] of Kashiwara and Schapira. We assume from now on that all our spaces are Hausdorff. Let $f: X \rightarrow Y$ be an exact and relatively smooth morphism of $\log$ analytic spaces, where $Y$ is fine and $X$ is relatively coherent. It follows from Theorem 3.7 that the fiber dimension of $f_{\log }: X_{\log } \rightarrow Y_{\log }$ is a locally constant function on $X_{\log }$, whose values are always even. Let us denote this dimension by $2 d_{X / Y}$.

Theorem 5.10 Let $f: X \rightarrow Y$ be an exact and relatively smooth morphism of $\log$ analytic spaces, where $Y$ is fine and $X$ is relatively coherent. Let $j: X^{v} \rightarrow X$ be the inclusion of the vertical locus of $f$ and let $f^{v}:=f \circ j$.

(1) The functor

$$
R f_{\log !}: D^{+}\left(X_{\log }\right) \rightarrow D^{+}\left(Y_{\log }\right)
$$

admits a right adjoint $R f_{\log }^{!}$, and for any $G \in D^{+}\left(Y_{\log }\right)$,

$$
R f_{\log }^{!} G \cong f_{\log }^{-1} G \otimes R f_{\log }^{!} \mathbf{Z} .
$$

(2) There is a canonical isomorphism

$$
R f_{\log }^{!} \mathbf{Z} \cong j_{!}^{\log }\left(\mathbf{Z}_{X_{\log }^{v}}\left[2 d_{X / Y}\right]\right)
$$

uniquely determined by the fact that its restriction to each of the fibers of a coherent small locus of $f_{\log }$ (see Remark 5.9) is the canonical one described in Proposition 5.8. Consequently, for any $G$

$$
R f_{\log }^{!} G \cong j_{!}^{\log } f_{\log }^{v^{-1}} G\left[2 d_{X / Y}\right] .
$$

(3) Let $F$ be an object of $D^{+}\left(X_{\log }\right)$ and $G$ be an object of $D^{+}\left(Y_{\log }\right)$. Then there is a natural isomorphism

$$
R \mathcal{H o m}\left(R f_{\log !} F, G\right) \cong R f_{\log *} R \mathcal{H o m}\left(F, j_{!}^{\log } f_{\log }^{v^{-1}} G\left[2 d_{X / Y}\right]\right) .
$$

In particular, if $f$ is proper and $d_{X / Y}$ is a constant $d$,

$$
\mathcal{H o m}\left(R^{q} f_{\log *} \mathbf{Q}, \mathbf{Q}\right) \cong R^{2 d-q} f_{\log !}^{v} \mathbf{Q} .
$$

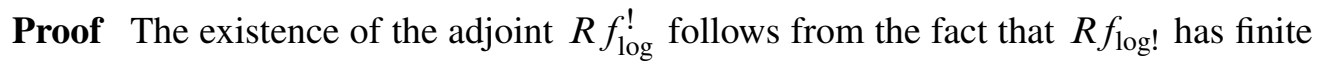
cohomological dimension, as explained by Kashiwara and Schapira [10, 3.1.5]. This finiteness follows in turn from the fact that the fibers of $f_{\log }$ are manifolds with 
boundary; see Iversen [8, III Section 9]. The formula for $R f_{\log }^{!}$in (1) follows from the fact that $f$ is a topological submersion; see Kashiwara and Schapira [10,3.3.2] and Verdier [21, 5.1]. Statement (3) follows immediately from Verdier duality [10, 3.1.10] and (2), which is the main difficulty.

We prepare for the proof of (2) with the following lemma.

Lemma 5.11 Consider a Cartesian square

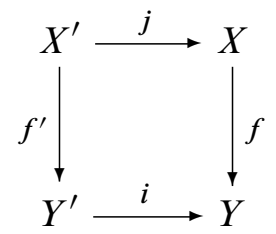

of locally compact Hausdorff spaces. Assume that $R f_{\text {! }}$ has finite cohomological dimension, so that $R f^{!}$exists [10,3.1.5].

(1) $R f_{!}^{\prime}$ has finite cohomological dimension, and there is a natural map

$$
j^{-1} \circ R f^{!} \rightarrow R f^{\prime !} \circ i^{-1}
$$

(2) This map is an isomorphism if $f$ is a topological submersion and $Y$ and $Y^{\prime}$ are locally connected.

(3) If $Y$ is locally connected and $f$ is a topological submersion whose fibers are manifolds of dimension $n$, then $R f^{!}\left(\mathbf{Z}_{Y}\right)$ is locally isomorphic to $\mathbf{Z}_{X}[n]$, and its formation commutes with base change.

Proof Statement (1) is from Kashiwara and Schapira [10, 3.1.9]. Statement (2) is a local problem so we may assume that $f$ is a projection mapping $Y \times Z \rightarrow Y$. Then the square in the lemma becomes identified with the left square of the following diagram:

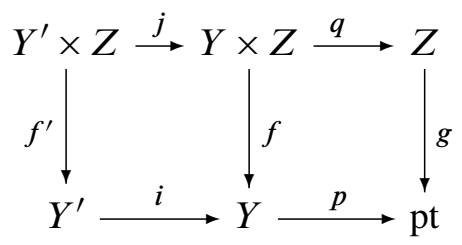

Let $q^{\prime}:=q \circ j$ and $p^{\prime}:=p \circ i$. Then there is a commutative diagram

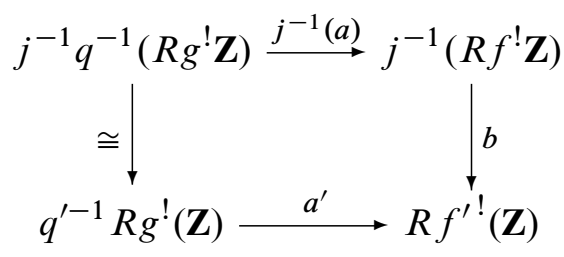


in which $a$ is the base change map for the square on the right, $a^{\prime}$ is the base change map for the outer rectangle, and $b$ is the base change map for the square on the left. Thus we are reduced to proving that $a$ and $a^{\prime}$ are isomorphisms. It suffices to treat $a$, corresponding to the case of the square on the right. This statement (under a different hypothesis) is asserted without proof in Verdier [21, Section 5]. To prove it in our situation, let $K_{Z}^{*}$ be the Godement resolution of the constant sheaf $\mathbf{Z}_{Z}$ on $Z$. This is a complex of $Z$-soft and flat sheaves, and hence for any open set $V$ of $Z, \operatorname{Rg}^{!}(\mathbf{Z})(V)=\operatorname{Hom}\left(g_{!}\left(K_{V}^{*}\right), \mathbf{Z}\right)$. On the other hand, $q^{-1}\left(K_{Z}^{\cdot}\right)$ is an $f$-soft and flat resolution of $\mathbf{Z}_{Y \times Z}$, and hence if $U$ is an open set in $Y, R f^{!}(\mathbf{Z})(U \times V)=$ $\operatorname{Hom}\left(f_{!}\left(q^{-1} K_{Z}^{\cdot}\right)_{U \times V}, \mathbf{Z}\right)$. If $V=Z$ and $U=Y$, the proper base change theorem says that the natural map $p^{-1} g_{!}\left(K_{Z}^{\cdot}\right) \rightarrow f_{!}\left(q^{-1} K_{Z}^{\cdot}\right)$ is an isomorphism. Here $K^{\cdot}:=$ $g_{!}\left(K_{Z}^{*}\right)$ is a complex of abelian groups, and so $p^{-1} g_{!}\left(K_{Z}^{\cdot}\right)$ is a constant complex of sheaves on $Y$. If $U$ is any connected open subset of $Y, p^{-1} g_{!}\left(K_{Z}^{\cdot}\right)(U)=K^{\cdot}$, and since $Y$ is locally connected, it follows that, if $Y$ is also connected,

$$
\operatorname{Hom}_{Y}\left(p^{-1} g_{!}\left(K_{Z}^{\cdot}\right), \mathbf{Z}\right)=\operatorname{Hom}\left(K^{*}, \mathbf{Z}\right)=\Gamma\left(Z, R g^{!}(\mathbf{Z})\right) .
$$

In other words, the natural map

$$
\Gamma\left(Z, R g^{!}(\mathbf{Z})\right) \rightarrow \Gamma\left(Y \times Z, R f^{!}(\mathbf{Z})\right)
$$

is an isomorphism if $Y$ is connected. Now if $z \in Z$ and $y \in Y$ and we take the limit over all connected neighborhoods of $y$ and all neighborhoods of $z$, we see that the stalk of the base change map $q^{-1} R g^{!}(\mathbf{Z}) \rightarrow R f^{!}(\mathbf{Z})$ is an isomorphism, as required. Statement (3) follows from (2) and the fact that $R f^{!}(\mathbf{Z})=\mathbf{Z}[n]$ when $f$ is the projection from $\mathbf{R}^{n}$ to a point.

In the situation of (3) of Lemma 5.11, the sheaf $o(f):=\mathcal{H}^{-n}\left(R f^{!}(\mathbf{Z})\right)$ is called the relative orientation sheaf of the morphism $f$. In particular, if $f$ is the projection from a smooth manifold $M$ to a point, $o(f)$ is the usual orientation sheaf of $M$. By part (2) of Lemma 5.11, if $f$ is a topological submersion whose fibers are manifolds, then, at least over a locally connected base, the restriction of $o(f)$ to a fiber is just the orientation sheaf of the fiber. We conclude from this the following fact, which is surely well-known.

Corollary 5.12 Let $f: X \rightarrow Y$ be a smooth morphism of complex analytic spaces, of relative dimension $d$. Then the relative orientation sheaf $o(f)$ is constant, and admits a unique trivialization whose restriction to the fibers is the canonical orientation coming from its structure as a complex manifold.

Let us return to the situation of the theorem. Our goal is to prove that $o\left(f_{\log }\right)$ is constant. Let us first consider the case when $f$ is given by a morphism of monoids $\theta: P \rightarrow Q$. 
Note that without loss of generality we may work everywhere with saturated monoids, since saturating a log space $X$ does not change $X_{\log }$.

Lemma 5.13 Let $\theta: P \rightarrow Q$ be a homomorphism of fine saturated monoids. Assume that $P$ is sharp and $Q$ is torsion free and that $\theta$ is local and locally exact. Let $\mathcal{M}_{Q}$ denote the usual $\log$ structure on $A_{Q}$, let $\mathcal{F} \subseteq \mathcal{M}_{Q}$ denote the sheaf of faces generated by $P$, and assume that the stalks of $\mathcal{M}_{Q} / \mathcal{F}$ are free. Then the relative orientation sheaf $o\left(A_{\theta}^{\log }(\mathcal{F})\right)$ is constant. In fact there is a unique global generator of $o\left(A_{\theta}^{\log }(\mathcal{F})\right)$ whose restriction to each of the fibers of a small locus of $A_{Q}^{\log }(\mathcal{F}) \rightarrow A_{P}^{\log }$ induces the orientation coming from the complex analytic structure constructed in Proposition 5.8.

Proof Choose a positive integer $n$, and consider the diagram

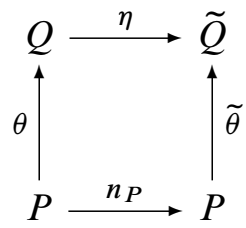

where $\widetilde{Q}$ is the pushout in the category of saturated monoids. By Theorem (A.3.4) and Tsuji's theorem (A.4.2) of [7], there exists a choice of $n$ such that the morphism $\tilde{\theta}$ is saturated. It follows then that the map $P^{\mathrm{gp}} \rightarrow \widetilde{Q}^{\mathrm{gp}}$ admits a section [7, A.4.1].

Let $X(\mathcal{M})(\operatorname{resp} . \tilde{X}(\mathcal{M}))$ denote $A_{Q}\left(\operatorname{resp} . A_{\widetilde{Q}}\right)$ with the $\log$ structure $\mathcal{M}_{Q}\left(\underset{\widetilde{F}}{\operatorname{resp} .} \mathcal{M}_{\tilde{Q}}\right)$ and let $Y$ be $A_{P}$ with the $\log$ structure coming from $P \rightarrow A_{P}$. Let $\widetilde{\mathcal{F}}$ denote the sheaf of faces in $\mathcal{M}_{\tilde{Q}}$ generated by $P$ and let $X(\mathcal{F})$ (resp. $\widetilde{X}(\mathcal{F})$ ) denote $A_{Q}$ (resp. $A_{\tilde{Q}}$ ) with the log structure $\mathcal{F}$ (resp. $\tilde{\mathcal{F}}$ ). Let $\zeta: Y \rightarrow Y$ (resp. $f: X(\mathcal{F}) \rightarrow Y$, $\tilde{f}: \tilde{X}(\mathcal{F}) \rightarrow Y$ ) be the map induced by $A_{n_{P}}$ (resp. $A_{\theta}, A_{\tilde{\theta}}$ ). Let $X^{\text {sm }}$ denote the open subset of $X$ where $X(\mathcal{M}) \rightarrow Y$ is small. Recall from Remark 5.9 that $X^{\mathrm{sm}}(\mathcal{F}) \rightarrow Y$ is also small and that $\mathcal{F}=\mathcal{M}$ on $X^{\text {sm }}$. Then we have the following commutative diagrams:
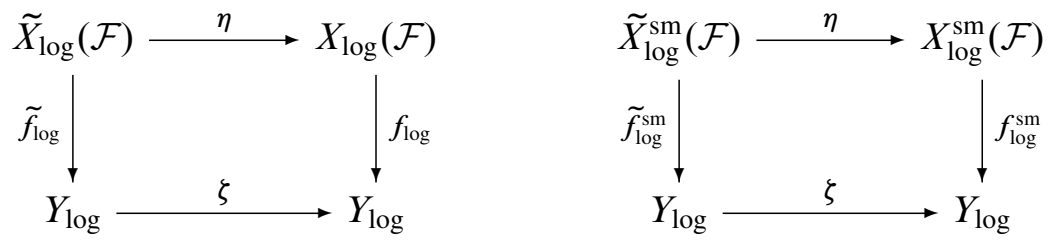

The map $\tilde{X}_{\log }(\mathcal{M}) \rightarrow X_{\log }(\mathcal{M})$ is proper and surjective, by [9, 5.1, 5.1.1], and it follows that the same is true for the map $\eta$ in the first diagram above. Since $\tilde{f}^{\text {sm }}$ is 
saturated and small, it is strict [7, A.4.1], and it follows that the diagram

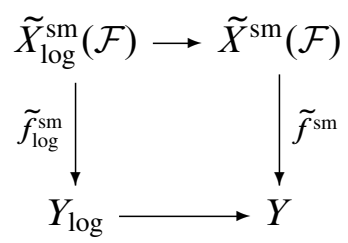

is Cartesian. Since $\tilde{f}^{\mathrm{sm}}$ is strict and smooth, it is a smooth map of complex analytic varieties without the log structures. By Corollary 5.12, its relative orientation sheaf $o\left(\tilde{f}^{\mathrm{sm}}\right)$ is constant and admits a unique trivialization $\tilde{\gamma}^{\mathrm{sm}}$ compatible with the orientations of the fibers coming from their complex analytic structures. By base change, $\tilde{\gamma}^{\mathrm{sm}}$ induces a trivialization of $o\left(\tilde{f}_{\log }^{\mathrm{sm}}\right)$ with the same property and which we denote by the same symbol.

As we saw in the proof of Theorem 3.7, the choice of an effective generating cycle for $C_{\tilde{Q}}$ defines a homeomorphism

$$
\tilde{X}_{\log }(\mathcal{F}) \cong Y_{\log } \times A_{\widetilde{Q}, P}^{\log }(\mathcal{F}) \cong Y_{\log } \times A_{\widetilde{Q} / P}
$$

sending $\tilde{f}_{\text {log }}$ to the projection mapping, where $A_{\widetilde{Q} / P}$ is a complex manifold. It follows from Lemma 5.11 that $o\left(\tilde{f}_{\text {log }}\right)$ is constant, that is, isomorphic to the constant sheaf $\mathbf{Z}$ on $\tilde{X}_{\log }(\mathcal{F})$. (Warning: as far as we know, the isomorphism given by the complex structure may depend on the choice of the effective generating cycle.) Consider the open subset

$$
\tilde{f}_{\log }^{-1}\left(Y^{*}\right)=\tilde{X}_{\log }^{*}(\mathcal{F}) \cong A_{\tilde{Q}_{P}}
$$

of $\tilde{X}_{\log }(\mathcal{F})$. Then $\widetilde{Q}_{P}^{*}=\langle P\rangle^{\mathrm{gp}}$, and since $\widetilde{Q}$ is saturated, the torsion subgroup of $\widetilde{Q}^{\mathrm{gp}}$, and in particular of $\langle P\rangle^{\mathrm{gp}}$, is contained in $\widetilde{Q}^{*}$. Since $Y_{\log }$ is connected, the connected components of $\tilde{X}_{\log }(\mathcal{F})$ are identified with those of $A_{\widetilde{Q} / P}$ and hence with the torsion subgroup of $\widetilde{Q} / P$. Since $P \rightarrow \widetilde{Q}$ is a saturated morphism, [7, A.4.1] implies that $\widetilde{Q}^{\mathrm{gp}} / P^{\mathrm{gp}}$ is torsion free. Thus the connected components of $\tilde{X}_{\log }(\mathcal{F})$ and of $\tilde{X}_{\log }^{*}(\mathcal{F})$ are both identified with the torsion subgroup of $\widetilde{Q}$, and hence the natural map

$$
H^{0}\left(\tilde{X}_{\log }(\mathcal{F}), \mathbf{Z}\right) \rightarrow H^{0}\left(\tilde{X}_{\log }^{*}(\mathcal{F}), \mathbf{Z}\right)
$$

is an isomorphism. On the other hand, $\tilde{X}_{\log }^{*}(\mathcal{F})$ is contained in $\tilde{X}_{\log }^{\mathrm{sm}}(\mathcal{F})$, so we have a natural global section $\tilde{\gamma}^{\mathrm{sm}}$ of the (constant) sheaf $o\left(\tilde{f}_{\log }\right)$ on $\tilde{X}_{\log }^{*}(\mathcal{F})$. Thus by the isomorphism above, this global section extends uniquely to a global section $\tilde{\gamma}$ of $o(\tilde{f})$ on all of $\tilde{X}_{\log }(\mathcal{F})$.

We claim that $\tilde{\gamma}$ descends to a trivialization $\gamma$ of $o\left(f_{\log }\right)$. The restriction of $\tilde{\gamma}$ to $\tilde{X}^{*}$ descends to $X^{*}$, since $f^{*}: X^{*} \rightarrow Y^{*}$ is a smooth map of smooth complex manifolds. It follows that the two pullbacks of $\tilde{\gamma}$ to $\tilde{X}_{\log }(\mathcal{F}) \times_{X_{\log }(\mathcal{F})} \tilde{X}_{\log }(\mathcal{F})$ agree on $\tilde{X}^{*} \times_{X^{*}} \tilde{X}^{*}$, 
and since this set is dense, they agree everywhere. Since $\eta$ is a finite surjective map, proper descent (see for example [1, 4.1.6]) implies that $\tilde{\gamma}$ descends to a trivialization $\gamma$ of $o\left(f_{\log }\right)$ on $X_{\log }(\mathcal{F})$, as required. Since $\tilde{\gamma}^{\text {sm }}$ is compatible with the complex analytic structure of the fibers of $f_{\log }^{\mathrm{sm}}$, the same is true of $\gamma^{\mathrm{sm}}$.

Now we prove (2) of Theorem 5.10 with the assumption that $f$ is vertical. Since in this case $f_{\log }$ is a submersion whose fibers are manifolds, $o\left(f_{\log }\right)$ is defined and locally isomorphic to $\mathbf{Z}$. Locally on $X, f$ admits a chart, and hence by Lemma 5.13, $X$ admits an open covering on which there exist isomorphisms as in (2). Since the small locus is dense in every fiber, these local isomorphisms agree, and hence patch to a unique isomorphism on all of $X_{\log }$.

For the general case, let $X^{v}$ be the vertical locus of $f$, and recall from Theorem 3.7 that the pair $\left(X_{\log }, X_{\log }^{v}\right)$ is locally isomorphic to $Y_{\log } \times\left(M, M^{*}\right)$ where $M$ is a manifold with boundary $M \backslash M^{*}$. Then the vertical case gives an isomorphism $\mathbf{Z}\left[2 d_{X / Y}\right] \cong j_{\log }^{-1} R f_{\log }^{!}(\mathbf{Z})$ and hence a map

$$
j_{\log !} \mathbf{Z}\left[2 d_{X / Y}\right] \rightarrow R f_{\log }^{!}(\mathbf{Z}) .
$$

Since both sides commute with base change, it is enough to check that this map is an isomorphism along the fibers. Then the result follows from the standard computation of the dualizing complex of a manifold with boundary; see Iversen [8, V, Example 2.9].

Corollary 5.14 With the hypotheses of Theorem 5.10, the fibers of $f_{\log }$ are orientable manifolds with boundary.

\section{Appendix: Local constancy of sheaves}

The purpose of this appendix is to give an elementary proof, based on the introductory discussion of vanishing cycles in SGA 7 [5, Exposé 1], of the following basic result.

Theorem 6.1 Let $f: X \rightarrow S$ be a proper separated submersion and let $F$ be a locally constant sheaf of abelian groups on $X$. Assume that $S$ is locally path connected and semilocally simply connected (see for example the textbook of Greenberg [3, II, Section 6]). Then for each $q \in \mathbf{Z}, R^{q} f_{*} F$ is locally constant on $S$.

Proof First we shall prove this when $S$ is the unit interval. This case relies on the following criterion. 
Proposition 6.2 Let $F$ be a sheaf of abelian groups on the unit interval $I$. For $s, t$ with $0 \leq s<t \leq 1$, let $J:=[s, t]$, let $F_{J}$ be the restriction of $F$ to $J$, and let $j:[s, t) \rightarrow[s, t]$ and $j^{\prime}:(s, t] \rightarrow[s, t]$ be the inclusions. Suppose that for every such $s$ and $t$, the maps

(1) $F_{J} \rightarrow j_{*} j^{*} F_{J}$,

(2) $F_{J} \rightarrow j_{*}^{\prime} j^{\prime *} F_{J}$

are isomorphisms. Then $F$ is constant.

Proof Suppose that the maps (1) are all isomorphisms. We claim first that for every $t^{\prime}>t>s$, the map $F\left(\left[s, t^{\prime}\right)\right) \rightarrow F([s, t))$ is injective. Suppose $f$ is a section of $F\left(\left[s, t^{\prime}\right)\right)$ which vanishes on $[s, t)$. Let $T$ be the set of all $t^{\prime \prime}$ such that $f$ vanishes on $\left[s, t^{\prime \prime}\right)$. Evidently $t \in T$. Let $b$ be the supremum of $T$. For every $b^{\prime}<b$, there is some $t^{\prime \prime} \in T$ such that $t^{\prime \prime}>b^{\prime}$. Then $f$ vanishes on $\left[s, t^{\prime \prime}\right)$ and hence also on $\left[s, b^{\prime}\right)$. Since the set of all $\left[s, b^{\prime}\right)$ with $b^{\prime}<b$ covers $[s, b)$, it follows that $b \in T$. Say $b<t^{\prime}$. Let $J:=[s, b]$, and observe that since $F_{J} \rightarrow j_{*} j^{*} F_{J}$ is an isomorphism, $f$ also vanishes on $[s, b]$, and hence in some neighborhood of $b$. This contradicts the fact that $b$ is an upper bound for $T$. Hence $b=t^{\prime}$, proving the injectivity. Next we claim that for every $t \geq s$ the map $F([s, 1]) \rightarrow F([s, t])$ is surjective. Suppose $f$ is a section of $F$ over $[s, t]$. We may assume $t<1$. Hence there exist some $t^{\prime}>t$ and $f^{\prime} \in F\left(\left[s, t^{\prime}\right)\right)$ such that $f^{\prime}$ extends $f$. We know $f^{\prime}$ is unique by the injectivity proved above. Let $T$ be the set of all $t^{\prime}$ for which there exists such an $f^{\prime}$ on $\left[s, t^{\prime}\right)$ and let $b$ be the supremum of $T$. Then for each $b^{\prime}<b$, there is some $t^{\prime}>b^{\prime}$ in $T$. Hence there is a (unique) $f^{\prime}$ on $\left[s, t^{\prime}\right)$ extending $f$. The set of all $\left[s, t^{\prime}\right)$ covers $[s, b)$ and by the uniqueness these $f^{\prime}$ patch to an extension of $f$ to $[s, b)$. Thus $b \in T$. The fact that (1) is an isomorphism then implies that $f^{\prime}$ extends to $[s, b]$. If $b=1$ we are done, and otherwise we find a contradiction.

We have checked that for every $s<t$, the map $F([s, 1]) \rightarrow F([s, t])$ is bijective. If in addition the maps in (2) are all isomorphisms, a similar argument proves that the map $F([0,1]) \rightarrow F([s, 1])$ is bijective. Then for every $s<t$, it follows that the maps $F([0,1]) \rightarrow F([s, t]) \rightarrow F((s, t))$ are bijective, and hence that $F$ is constant.

Lemma 6.3 Let $f: X \rightarrow I$ be a topological submersion, and let $F$ be a locally constant abelian sheaf on $X$. Let $X^{\prime}:=f^{-1}[0,1)$ and let $k: X^{\prime} \rightarrow X$ be the inclusion. Then the natural map $F \rightarrow R k_{*} k^{*} F$ is an isomorphism in $D^{+}(X)$. Hence for every $q \in \mathbf{Z}$, the natural map

$$
R^{q} f_{*} F \rightarrow R^{q}(f \circ k)_{*}\left(k^{*} F\right)
$$

is an isomorphism. 
Proof Let $Z$ be the complement of $X^{\prime}$ in $X$. It suffices to show that the cohomology sheaves $\mathcal{H}_{Z}^{q}(X, F)$ are all zero. This is a local condition on $X$, so we may assume that $F$ is constant and that $X=Y \times J$, where $J:=(r, 1]$ for some $r<1$. We claim that the map

$$
H^{q}(Y \times J, F) \rightarrow H^{q}\left(Y \times J^{\prime}, F\right)
$$

is an isomorphism, where $J^{\prime}:=(r, 1)$. Choose $r^{\prime} \in J$. Then the map sending $J$ (resp. $J^{\prime}$ ) to $r^{\prime}$, followed by the inclusion of $\left\{r^{\prime}\right\}$ in $J$ (resp. $J^{\prime}$ ) is homotopic to the identity. It follows that the inclusion of $Y \times J^{\prime}$ in $Y \times J$ is a homotopy isomorphism. Hence it induces an isomorphism on cohomology, by Kashiwara and Schapira [10, 2.7.5].

Lemma 6.4 Theorem 6.1 is true if $S=I$.

Proof We prove by induction on $q$ that $\mathcal{F}^{q}:=R^{q} f_{*} F$ is constant on $I$. Assume this is true for $q^{\prime}<q$. Let $j: I^{\prime}:=[0,1) \rightarrow I$ be the inclusion, let $X^{\prime}:=f^{-1}\left(I^{\prime}\right)$, and let $f^{\prime}: X^{\prime} \rightarrow I^{\prime}$ be the map induced by $f$. We have a commutative diagram:

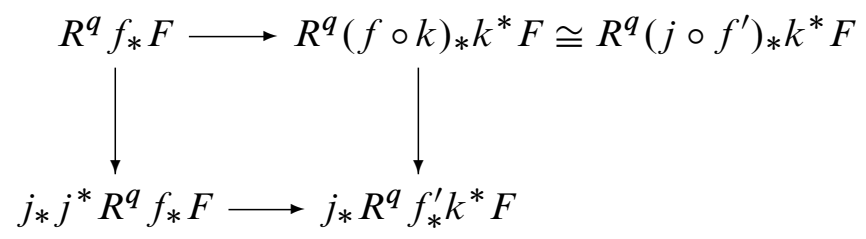

The top horizontal arrow is an isomorphism by the previous lemma. The horizontal arrow on the bottom is trivially an isomorphism. The vertical arrow on the right is the edge homomorphism associated with the spectral sequence with $E_{2}^{p, q^{\prime}}=R^{p} j_{*} R^{q^{\prime}} f_{*}^{\prime} F$. The induction hypothesis implies that $R^{q^{\prime}} f_{*}^{\prime} F$ is constant on $I^{\prime}$ if $q^{\prime}<q$ and hence that $E_{2}^{p, q^{\prime}}=0$ for $q^{\prime}<q$ and $p>0$. This implies that the right vertical arrow is an isomorphism. It follows that the left vertical arrow is also an isomorphism.

Now if $0 \leq s<t \leq 1$, let $J:=[s, t]$ and let $i: J \rightarrow I$ be the inclusion. Let $f_{J}: X_{J} \rightarrow J$ be the pullback of $f$ to $J$ and let $F_{J}$ be the pullback of $F$ to $X_{J}$. By the proper base change theorem, the natural map $i^{*} \mathcal{F}^{q} \rightarrow R^{q} f_{J *} F_{J}$ is an isomorphism for all $q$. The above argument applies equally to $f_{J}$, and it follows that the natural map $\mathcal{F}_{J}^{q}:=i^{*} \mathcal{F}^{q} \rightarrow j_{*} j^{*}\left(\mathcal{F}_{J}^{q}\right)$ is an isomorphism, as in Proposition 6.2. The statement for $j^{\prime}$ is proved similarly. Then it follows from Proposition 6.2 that $\mathcal{F}^{q}$ is constant.

Lemma 6.5 Let $S$ be a locally path connected and semilocally simply connected space, and let $\mathcal{F}$ be an abelian sheaf on $S$. Assume that for every path $\gamma: I \rightarrow S$, the pullback $\gamma^{*} \mathcal{F}$ is constant. Then $\mathcal{F}$ is locally constant. 
Proof First we prove this when $S=I \times I$. We show first that if $U$ is a path connected subset of $S$ and $x \in U$, the map $\mathcal{F}(U) \rightarrow \mathcal{F}_{x}$ is injective. Indeed, if $f$ is an element of the kernel and $y \in U$, there is a path $\gamma$ in $U$ from $x$ to $y$, and $\gamma^{*} \mathcal{F}$ is constant. It follows that the germ of $f$ at $y$ also vanishes, and since this is true for every $y, f$ vanishes.

Next we prove that the map $\mathcal{F}(S) \rightarrow \mathcal{F}_{x}$ is bijective, where $x:=(0,0)$. Choose $g \in \mathcal{F}_{x}$. Since the restriction of $\mathcal{F}$ to $I \times 0$ is constant, $g$ extends uniquely to a section of $\mathcal{F}$ on $I \times 0$. Let $T$ be the set of all $t$ such that $g$ extends to a section of $\mathcal{F}$ on $I \times[0, t]$, and let $b$ be the supremum of $T$. We claim that $b \in T$. If $b=0$ this is certainly true. If $b>0$, then for every $b^{\prime}<b$, there is a unique section of $\mathcal{F}$ on $I \times\left[0, b^{\prime}\right)$ extending $g$, and these patch to a section $f$ on $I \times[0, b)$. For each $a \in I$, the restriction of $\mathcal{F}$ to $a \times[0, b]$ is constant, so there is a unique section $f_{a}$ of $\mathcal{F}$ on $a \times[0, b]$ which agrees with $f$ on $a \times[0, b)$. Then there exist an open box $U$ around $(a, b)$ and a section $f_{U}$ of $\mathcal{F}$ on $U$ whose germ at $(a, b)$ agrees with the germ of $f_{a}$ at $(a, b)$. That is, for some $b^{\prime}<b, f_{U}$ and $f_{a}$ agree on $a \times\left(b^{\prime}, b\right]$. Then $V:=$ $U \cap I \times[0, b)$ is path connected, and the germs of $f_{U}$ and $f$ agree at the points of $V$ on $a \times\left(b^{\prime}, b\right)$. By the injectivity proved above, this implies that they agree on all of $V$ and hence patch to a global section on $I \times[0, b) \cup U$. Doing this for each $a$, we find a section of $\mathcal{F}$ on $I \times[0, b]$ whose germ at $x$ is $g$. This shows that $b \in T$. If $b<1$ we see from the compactness of $I \times[0, b]$ that $f$ extends to an open neighborhood of $I \times[0, b]$ in $I \times I[10,2.5 .2]$. But this would contradict the fact that $b$ is an upper bound for $T$. Thus $b=1$, and we are done.

It now follows easily that $\mathcal{F}$ is constant. Indeed, if $x^{\prime}$ is another point of $S$, there is a path $\gamma: I \rightarrow S$ joining $x$ and $x^{\prime}$, and since $\gamma^{*}(\mathcal{F})$ is constant, it follows that the natural map $\mathcal{F}(S) \rightarrow \mathcal{F}_{x^{\prime}}$ is also an isomorphism. This implies that $\mathcal{F}$ is constant.

Now suppose that $S$ is connected, simply connected and locally path connected. Given any two points $x$ and $y$ in $S$, there is a path $\gamma$ from $x$ to $y$, and hence a map $\phi_{y, x}$ from $\mathcal{F}_{x} \rightarrow \mathcal{F}_{y}$. Since $S$ is simply connected, any two paths are homotopic, and by the argument above, this isomorphism is independent of the choice of path. It follows that if $z$ is a third point of $S, \phi_{z, y} \phi_{y, x}=\phi_{z, x}$. Note that if $f$ is a section of $\mathcal{F}$ over some connected open subset $U$ of $S$ and $x$ and $y$ belong to $U$, then $\phi_{y, x}\left(f_{x}\right)=f_{y}$. Choose some $s \in S$. We claim that for each open subset $U$ of $S$ and each $f \in \mathcal{F}_{s}$, there is a unique $g \in \mathcal{F}(U)$ such that $g_{u}=\phi_{u, s}(f)$ for all $u \in U$. The uniqueness is clear. For the existence, consider pairs $\left(U^{\prime}, g^{\prime}\right)$ where $U^{\prime}$ is an open subset of $U$ and $g^{\prime} \in \mathcal{F}\left(U^{\prime}\right)$ is such that $g_{u^{\prime}}^{\prime}=\phi_{u^{\prime}, s}(f)$ for every $u^{\prime} \in U^{\prime}$. Order the set of such pairs as usual, and suppose that $(V, h)$ is maximal (by the sheaf axiom such a pair exists). Say $u \in U$, and choose a connected open neighborhood $U^{\prime}$ of $u$ and a $g^{\prime} \in \mathcal{F}\left(U^{\prime}\right)$ such that $g_{u}^{\prime}=\phi_{u, s}(f)$. If $u^{\prime}$ is any point of $U^{\prime}$, it follows that 
$g_{u^{\prime}}^{\prime}=\phi_{u^{\prime}, u}\left(g_{u}^{\prime}\right)=\phi_{u^{\prime}, u} \phi_{u, s}(f)=\phi_{u^{\prime}, s}(f)$. In particular, if $u^{\prime} \in U^{\prime} \cap V, g_{u^{\prime}}^{\prime}=h_{u^{\prime}}$. This implies that the restriction of $g^{\prime}$ to $U^{\prime} \cap V$ agrees with the restriction of $h$ to $U^{\prime} \cap V$. Then $h$ extends to $U^{\prime} \cup V$, so by maximality $U^{\prime} \subseteq V$. Hence $V=U$. Thus we have constructed, for every open $U$ in $S$, a map $\sigma_{U}: \mathcal{F}_{S} \rightarrow \mathcal{F}(U)$ such that $\sigma_{U}(f)_{u}=\phi_{u, s}(f)$ for all $u \in U$. It is clear that the maps $\mathcal{F}_{s} \rightarrow \mathcal{F}(U)$ are compatible with restriction, and hence define a map from the constant sheaf $\mathcal{F}_{S}$ to the sheaf $\mathcal{F}$. It follows from our assumption that this map is an isomorphism on stalks. Hence it is an isomorphism and $\mathcal{F}$ is constant.

Now we prove the general case. Since $S$ is locally path connected, its connected components are open and locally path connected, and it is enough to prove the result for each connected component. Since $S$ is semilocally simply connected, it has a universal cover $\widetilde{S} \rightarrow S$. By the previous case, the pullback of $\mathcal{F}$ to $\widetilde{S}$ is constant. Since $\widetilde{S} \rightarrow S$ is a covering space, it follows that $\mathcal{F}$ is locally constant.

Now we can finish the proof of Theorem 6.1. Let $\gamma: I \rightarrow S$ be a path. Then $f_{\gamma}:=$ $X \times{ }_{S} I \rightarrow I$ is a proper separated submersion, and by Lemma 6.4, the cohomology sheaves $R^{q} f_{\gamma_{*}} F$ are constant. By the proper base change theorem, $R^{q} f_{\gamma_{*}} F \cong \gamma^{*} \mathcal{F}^{q}$. Thus the result follows from Lemma 6.5.

\section{References}

[1] M Artin, A Grothendieck, J L Verdier, Théorie des topos et cohomologie étale des schémas. Tome 2, Lecture Notes in Math. 270, Springer, Berlin (1972) MR0354653 Séminaire de Géométrie Algébrique du Bois-Marie 1963-1964 (SGA 4), Avec la collaboration de N Bourbaki, P Deligne, B Saint-Donat

[2] W Fulton, Introduction to toric varieties, Annals of Math. Studies 131, Princeton Univ. Press (1993) MR1234037

[3] MJ Greenberg, Lectures on algebraic topology, W A Benjamin, New YorkAmsterdam (1967) MR0215295

[4] M Gross, B Siebert, Mirror symmetry via logarithmic degeneration data I, J. Differential Geom. 72 (2006) 169-338 MR2213573

[5] A Grothendieck, Groupes de monodromie en géométrie algébrique I, Lecture Notes in Math. 288, Springer, Berlin (1972) MR0354656 Séminaire de Géométrie Algébrique du Bois-Marie 1967-1969 (SGA 7 I), Avec la collaboration de M Raynaud, D S Rim

[6] H Hironaka, Triangulations of algebraic sets, from: "Algebraic geometry (Proc. Sympos. Pure Math., Vol. 29, Humboldt State Univ., Arcata, Calif., 1974)”, (R Hartshorne, editor), Amer. Math. Soc. (1975) 165-185 MR0374131 
[7] L Illusie, K Kato, C Nakayama, Quasi-unipotent logarithmic Riemann-Hilbert correspondences, J. Math. Sci. Univ. Tokyo 12 (2005) 1-66 MR2126784

[8] B Iversen, Cohomology of sheaves, Universitext, Springer, Berlin (1986) MR842190

[9] T Kajiwara, C Nakayama, Higher direct images of local systems in log Betti cohomology, J. Math. Sci. Univ. Tokyo 15 (2008) 291-323 MR2478113

[10] M Kashiwara, P Schapira, Sheaves on manifolds, Grund. der Math. Wissenschaften 292, Springer, Berlin (1990) MR1074006 With a chapter in French by C Houzel

[11] K Kato, Logarithmic structures of Fontaine-Illusie, from: "Algebraic analysis, geometry, and number theory (Baltimore, MD, 1988)", (J-I Igusa, editor), Johns Hopkins Univ. Press, Baltimore, MD (1989) 191-224 MR1463703

[12] K Kato, Toric singularities, Amer. J. Math. 116 (1994) 1073-1099 MR1296725

[13] K Kato, C Nakayama, Log Betti cohomology, log étale cohomology, and log de Rham cohomology of log schemes over C, Kodai Math. J. 22 (1999) 161-186 MR1700591

[14] A Ogus, Introduction to logarithmic algebraic geometry, in preparation

[15] A Ogus, Relatively coherent log structures, in preparation

[16] A Ogus, On the logarithmic Riemann-Hilbert correspondence, Doc. Math. (2003) 655-724 MR2046612 Kazuya Kato's fiftieth birthday

[17] L Pachter, B Sturmfels (editors), Algebraic statistics for computational biology, Cambridge Univ. Press (2005) MR2205865

[18] L C Siebenmann, Deformation of homeomorphisms on stratified sets. I, II, Comment. Math. Helv. 47 (1972) 123-136; ibid. 47 (1972), 137-163 MR0319207

[19] S Usui, Recovery of vanishing cycles by log geometry: case of several variables, from: "Commutative algebra, algebraic geometry, and computational methods (Hanoi, 1996)", (D Eisenbud, editor), Springer, Singapore (1999) 135-143 MR1714854

[20] S Usui, Recovery of vanishing cycles by log geometry, Tohoku Math. J. (2) 53 (2001) 1-36 MR1808639

[21] J-L Verdier, Dualité dans la cohomologie des espaces localement compacts, from: “Séminaire Bourbaki 1965/1966, Vol. 9, Exp. No. 300”, Soc. Math. France (1995) 337-349 MR1610971

Department of Mathematics, Tokyo Institute of Technology

Ookayama, Meguro-ku, Tokyo 152-8551, Japan

Department of Mathematics, University of California

Berkeley, CA 94720, USA

cnakayam@math.titech.ac.jp, ogus@math.berkeley.edu

http://www . math . berkeley . edu/ ogus

Proposed: Richard Thomas

Received: 11 March 2010

Seconded: Jim Bryan, Frances Kirwan

Revised: 23 August 2010 